\title{
Adenylosuccinate Lyase activity in the Purine recycling pathway is essential for developmental timing, germline maintenance and muscle integrity in $C$. elegans
}

Roxane Marsac1, Benoît Pinson', Christelle Saint-Marc1, María Olmedo2,3, Marta ArtalSanz'2, Bertrand Daignan-Fornier 1 and José-Eduardo Gomes 1,4

1 - Institut de Biochimie et Génétique Cellulaires, Université de Bordeaux and CNRS UMR5095 , 33077 Bordeaux cedex, France

2 - Andalusian Center for Developmental Biology, Consejo Superior de Investigaciones Científicas/Junta de Andalucía/Universidad Pablo de Olavide, Department of Molecular Biology and Biochemical Engineering. 41013, Seville, Spain

3 - Current address: Department of Genetics, University of Seville, 41012, Seville, Spain

4 - Correspondence: jose-eduardo.gomes@u-bordeaux.fr

\section{Abstract}

Purine homeostasis is ensured through a metabolic network widely conserved from prokaryotes to humans. Purines can either be synthesized de novo, reused, or produced by interconversion of extant metabolites using the so-called recycling pathway. Although thoroughly characterized in microorganisms, such as yeast or bacteria, little is known about the regulation of this biosynthesis network in metazoans. In humans, several diseases are linked to purine biosynthesis deficiencies through yet poorly understood etiologies. Particularly, the deficiency in Adenylosuccinate Lyase (ADSL), one enzyme involved both in the purine de novo and recycling pathways, causes severe muscular and neuronal symptoms. In order to address the mechanisms underlying this deficiency, we established Caenorhabditis elegans as a metazoan model organism to study purine metabolism, while focusing on ADSL. We show that the purine biosynthesis network is functionally conserved in C. elegans. Moreover, ADSL is required for developmental timing and germline stem cell maintenance, and muscle integrity. Our results allow to ascribe developmental and tissue specific phenotypes to separable steps of the purine metabolic network in an animal model. Particularly, the muscle, germline and developmental defects are linked specifically to the ADSL role in the purine recycling pathway.

\section{Introduction}

Purine biosynthesis pathways ensure the production and homeostasis of AMP and GMP in the cell, and are widely conserved throughout evolution. The de novo pathway leads to the synthesis of IMP (Inosine monophosphate) using PRPP (5-Phosphoribosyl-1pyrophosphate) as precursor. The recycling pathway (a.k.a. salvage pathway) can then transform extant purines, including IMP synthesized through the de novo pathway, to produce AMP and GMP (Figure 1A, reviewed in [1]). Importantly, if available in the extracellular environment, purines can be recovered by specific transporters and subsequently be metabolized through the recycling pathway, which requires much less energy expenditure. Indeed, purine synthesis through the de novo pathway requires the hydrolysis of six more molecules of ATP, than purine synthesis through the recycling pathway.

Imbalance in purine metabolism is involved in several diseases that have been extensively characterized, most of them associated with mutations in genes encoding for purine biosynthesis enzymes (reviewed in [2,3]). The genetics and biochemistry of the 
purine biosynthesis are well established in unicellular organisms like bacteria or yeast, and are highly conserved. Nonetheless, how the disease-causing enzymatic deficiencies lead to the symptoms observed in patients remains elusive. It is particularly challenging to untangle the contribution of shortage in the final products - AMP and GMP - from mutationcaused accumulation of intermediate metabolites in the pathway. For instance, ZMP (5amino-4-imidazole carboxamide ribonucleotide monophosphate [4]) and SZMP (succinylZMP), two intermediates of the purine de novo pathway (Figure 1), are known to act as signal metabolites regulating gene expression transcriptionally in yeast [5]. Moreover, ZMP is known to activate AMPK (AMP activated kinase) by mimicking AMP [6] and SZMP has been shown to directly bind and interfere with pyruvate kinase activity in cancer cells [7]. In addition, SAMP (succinyl-AMP), an intermediate metabolite in the recycling pathway, has been shown to stimulate Insulin secretion in pancreatic cells [8]. Hence SZMP, ZMP or SAMP buildup could potentially disrupt homeostasis upon deficiencies in the enzymes using them as substrates - ADSL (SZMP and SAMP) and ATIC (ZMP, Figure 1A). Accordingly, mutations in the human genes encoding for these two enzymes are associated with ADSL deficiency and ATIC deficiency (a.k.a. AICAribosiduria), syndromes whose etiology remains unknown [9-14]. Adding to the complexity of the issue, ADSL catalyzes two different reactions, one in the de novo and one in the recycling pathway (Figure 1A), raising the question of which of the pathways is associated with the ADSL deficiency phenotypes.

The study of purine metabolic deficiencies in patients, faces yet other challenges, in comparison with microorganisms. One such challenge is tissue specificity, as different tissues may require different levels of purines, depending on their specialized function. For instance, in ADSL-deficiency patients, the most severe symptoms affect the muscles and the nervous system, suggesting these tissues have a larger requirement for purine metabolism, either for their function or during development. Another challenge is purine transport across tissues; the tissue affected by the deficiency not being necessarily the tissue where the enzyme is active. Moreover, purines can be available exogenously from the food source, therefore the consequences of purine metabolism deficiencies are not necessarily caused by limiting amounts of purines. Finally, purine metabolism might be subject to different regulations throughout development, in which case the impairments observed could be the consequence of a requirement for purine regulation at a specific developmental stage.

In order to address these issues, we sought to take advantage of $C$. elegans as model organism. Through a genetic approach, we characterized deficiencies in the purine biosynthesis pathways, focusing our analysis on an ADSL deficient mutant. Our goal was to establish the phenotypes associated with this enzymatic deficiency in order to uncover what functions, tissues and developmental stages are affected. Given ADSL is required both in the de novo and recycling pathways, we performed a comparative analysis with ATIC and GPPAT deficiencies to dissect the contribution of the de novo vs. recycling pathways to the phenotypes observed. We established that ADSL deficiency strongly disrupts muscle integrity, germline stem cells (GSCs) maintenance and post-embryonic development through the recycling pathway. By contrast, we found no essential requirement for the de novo pathway in $C$. elegans under standard laboratory conditions.

\section{Results}

- Purine biosynthesis pathway is functionally conserved in $C$. elegans 
In order to establish $C$. elegans as a model to study purine metabolism, we first sought to investigate the conservation of the purine biosynthesis pathways. We identified, through sequence homology, genes encoding the putative nematode enzymes involved in purine biosynthesis (Supplementary Figure 1A). We found that both de novo and recycling pathways are conserved in $C$. elegans, judged by sequence similarity. Specifically, for each enzyme we identified one single orthologue, in all but one case (three genes putatively encoding Xanthine Oxidase). Noteworthy, in S. cerevisiae the histidine pathway contributes to the purine de novo pathway, ZMP being a byproduct of histidine synthesis (reviewed in [1]). We have found no $C$. elegans orthologues for yeast HIS1, HIS4, HIS6 and HIS7 - required for ZMP synthesis through the histidine pathway - consistent with the histidine pathway loss in worms previously reported [15], thus ZMP is most likely synthesized only through the purine de novo pathway.

Given the sequence conservation, we undertook a functional analysis focusing on the ADSL enzyme, and including ATIC and GPPAT for comparison (see below). The genes encoding ADSL, ATIC and GPPAT in C. elegans were named respectively ads/-1, atic-1 and ppat-1 (wormbase.org). We first tested whether those three $C$. elegans enzymes were functionally conserved using an heterologous rescue assay. We transformed yeast Saccharomyces cerevisiae ade $13 \Delta$, ade16 $\Delta$ ade $17 \Delta$ and ade $4 \Delta$ mutants with plasmids containing the wild-type sequences of the predicted $C$. elegans homologous genes: adsl-1, atic-1 and ppat-1, respectively. Expression of adsl-1 restored adenine prototrophy of yeast ade13 $\Delta$ (Figure 1B), likewise expression of splicing isoform atic-1b restored adenine prototrophy of ade16 $\Delta$ ade17 $\Delta$ double mutant (Figure 1B). These results confirm adsl-1 encodes the ADSL enzyme and atic-1 encodes for the ATIC enzyme. By contrast, expression of ppat-1 alone was not sufficient to restore adenine prototrophy of yeast ade $4 \Delta$ (Figure 1B), thus in our heterologous rescue assay we could neither confirm nor rule out ppat-1 encodes the enzyme GPPAT.

We next tested functional conservation of ADSL, ATIC and GPPAT activities in vivo. For our functional analysis, we used deletion mutant alleles henceforth referred to as $\operatorname{adsl-1}(\Delta)$, atic-1 $(\Delta)$ and ppat-1( $\Delta)$ (see Materials and Methods). One would predict accumulation of ADSL substrates SZMP and SAMP upon adsl-1 loss-of-function as well as ZMP buildup in atic-1 $(\Delta)$, and no detectable de novo pathway intermediate metabolites in ppat-1 $(\Delta)$ (Supplementary Figure 1B). To test these hypotheses, we performed worm metabolic profiling by HPLC (Supplementary Figure 1C). Importantly, sequence alignment indicates ADSL, ATIC and GPPAT are highly conserved (Supplementary Figure 2). We observe in our metabolic profiling a consistent, accumulation of SZMP, and its riboside form SAICAR, upon adsl-1(RNAi), as well as a robust accumulation of SAMP, and its riboside form SAdo (Figure $1 \mathrm{C}$ ), while neither metabolite was detected in $\mathrm{N} 2$ reference strain (henceforth wild-type), demonstrating that both de novo and recycling pathways are functionally conserved in $C$. elegans, and adsl-1 is required for ADSL activity in vivo. Furthermore, we observe a modest but consistent accumulation of ZMP both in atic-1 $(\Delta)$ mutants (Figure 1D), as well as atic-1(RNAi) animals (not shown), demonstrating atic-1 encodes ATIC enzyme. The accumulation of SZMP and ZMP in adsl-1(RNAi) and atic-1( $\Delta)$ was rather modest, by contrast we observed a robust accumulation of SAMP in adsl-1(RNAi). These results suggests that $C$. elegans purine biosynthesis relies mostly on the recycling pathway, at least in standard culture conditions. Noteworthy, ATP levels are slightly lower in adsl-1(RNAi) compare to wild-type (Figure 1C), otherwise the abundance of ATP and GTP were not severely affected in the deficiencies analyzed. 
As to the conservation of GPPAT, the HPLC analysis provided crucial functional evidence: neither SZMP was detected in ppat-1(L); adsl-1(RNAi) (Figure 1C) nor was ZMP detected in ppat-1( $\Delta)$; atic-1( $\Delta)$ (Figure 1D), or ppat-1( $\Delta)$; atic-1(RNAi) (not shown). This demonstrates that ppat-1 gene is indeed required for purine synthesis through the de novo pathway. Noteworthy, SAMP accumulation in ppat-1(L); adsl-1(RNAi) is similar to adsl-1(RNAi) alone, providing an internal control for RNAi efficiency and further confirming SZMP accumulation in adsl-1(RNAi) is dependent on ppat-1. In summary, our metabolic profiling validates functional conservation of ADSL and ATIC, and demonstrates ppat-1 is required for the de novo pathway, consistent with encoding the GPPAT enzyme in $C$. elegans.

- Functional analysis of adsl-1

Because SZMP and SAMP are known to function as regulatory metabolites in other organisms $[5,8,16,17]$ and because they may play an important role in the etiology of the ADSL deficiency - the enzyme metabolizing these two compounds, we focused our functional analysis on ads/-1. In order to distinguish the effects of SZMP buildup from those due to the loss of de novo purine synthesis, we further include ppat-1 in our analysis. Moreover, in order to distinguish the effect of ADSL activity in the de novo pathway from the activity in the salvage pathway we used for comparison ATIC deficient worms. ATIC participates only in the de novo pathway and accumulates ZMP, which shares some targets with SZMP at least in yeast $[5,16]$. Finally, in order to test whether phenotypes observed in adsl-1( $\Delta$ ) were due to SZMP buildup, we performed systematically epistasis analysis in the ppat-1( $\Delta)$; adsl-1( $\Delta)$ double mutant, and similarly to test whether phenotypes in the atic-1( $\Delta)$ mutant were caused by ZMP accumulation we performed epistasis in the ppat-1( $\Delta)$; atic-1( $\Delta)$ double mutant. Throughout the manuscript, we refer collectively as "purine mutants" to all three single mutant homozygous animals, plus the ppat-1 $(\Delta)$; adsl-1 $(\Delta)$ and ppat-1 $(\Delta)$; atic-1( $\Delta)$ double mutants; and as "de novo" pathway mutants to ppat-1 $(\Delta)$, atic-1 $(\Delta)$ single mutants and ppat-1( $\Delta)$; atic-1( $\Delta)$ double homozygous mutant animals.

- adsl-1 is required for germline stem cells' (GSCs) maintenance.

The adsl-1( $\Delta$ ) homozygous animals display striking morphological defects, they are noticeably smaller than wild-type, sterile and present a protruding vulva (Figure $2 \mathrm{~A})$. We observe a complete absence of oocytes and sperm cells in adsl-1( $\Delta)$ and ppat-1( $\Delta)$; adsl-1( $\Delta$ ) worms, only a reduced number of nuclei morphologically resembling the GSCs are detectable near the vulva, where the germline distal tip cell is found in wild-type animals (Supplementary Figure 3A). By contrast, the homozygous mutants in the de novo pathway are viable and fertile, and the overall morphology is similar to wild-type controls. To better characterize the GSCs phenotype, we used a reporter transgene expressing H2B::GFP fusion in the GSCs under the control of the pie-1 promoter [18]. While in ppat-1 $(\Delta)$ single mutants the germline is indistinguishable from wild-type, in ads/-1( $\Delta)$ and ppat-1 $(\Delta)$; adsl-1 $(\Delta)$ animals sterility is fully penetrant and the germline is barely detectable if at all (Figure 2B). Indeed we observed $52 \%$ of adsl-1 $(\Delta)(n=23)$ and ppat-1( $\Delta)$; adsl-1( $\Delta)$ animals $(n=25)$ had no detectable H2B::GFP, 44\% had 10 or less H2B::GFP positive nuclei, and $4 \%$ had barely above $10 \mathrm{H} 2 \mathrm{~B}:$ :GFP positive nuclei. Hence adsl-1 is required for proliferation and/or maintenance of the GSCs, independently of ppat-1. 
It has been shown that the sterility associated with a deficiency in the pyrimidine recycling pathway could be rescued by supplementation of the culture medium with pyrimidine nucleosides [19], we thus tested whether supplementation with purine nucleosides could rescue adsl-1( $\Delta$ ) sterility. When cultured with $20 \mathrm{mM}$ Adenosine (Ado in Figure $1 A)$ in the medium from hatching until adulthood, both $\operatorname{ads} /-1(\Delta)(n=55)$ and ppat-1( $\Delta)$; adsl-1 $(\Delta)(n=54)$ were sterile, much like the non-supplemented controls $(n=80$ and $\mathrm{n}=88$, respectively). As expected, $100 \mathrm{mM}$ Inosine (Ino in Figure 1A) supplementation had no effect in $\operatorname{adsl-1}(\Delta)(\mathrm{n}=28)$ nor in ppat-1; adsl-1( $\Delta)(\mathrm{n}=64)$ sterility. Under the same conditions wild-type (20 mM Adenosine $\mathrm{n}=86 ; 100 \mathrm{mM}$ Inosine $\mathrm{n}$ = 99) and ppat-1 $(\Delta)(20 \mathrm{mM}$ Adenosine $\mathrm{n}=76 ; 100 \mathrm{mM}$ Inosine $\mathrm{n}=100)$ worms were fertile, to the same extent as non-treated controls (wild-type $n=116$; ppat-1 $(\Delta) n=114$ ). We found no evidence that purine supplementation in the culture medium allows to recover the GSCs defects of adsl-1( $\Delta)$.

As mentioned above, the de novo pathway mutants present a germline similar to wild-type, in contrast with the complete sterility of adsl-1( $\Delta)$. In $C$. elegans, adult hermaphrodites reproduce through self-fertilization, and the number of progeny being modulated by food source and metabolic status [20, 21]. In order investigated whether the de novo pathway could impact the level of fertility, we measured total number of eggs laid by ppat-1( $\Delta)$, atic-1( $\Delta)$ and ppat-1( $\Delta)$; atic-1( $\Delta)$ hermaphrodites throughout their reproductive life. We observed that ppat-1( $\Delta)$ fertility is in the wild-type range, while atic-1( $\Delta$ ) fertility is significantly lower (Supplementary Figure 3B). Importantly, ppat-1( $\Delta$ ) is epistatic to atic-1 $(\Delta)$ regarding fertility, suggesting that ZMP accumulation, rather than de novo pathway impairment, might reduce fertility. To test this hypothesis we cultured ppat-1 $(\Delta)$; atic-1 $(\Delta)$ in presence of ZMP precursor AICAR $(0.1 \mathrm{mM})$ in the culture medium under this condition internal ZMP builds up (see below). We observed a reduced fertility in ppat-1 $(\Delta)$; atic-1 $(\Delta)$ treated with AICAR, compared to the control (Supplementary Figure $3 \mathrm{~B})$, consistent with our hypothesis. Furthermore, wild-type worms cultured with $0.5 \mathrm{mM}$ AICAR also displayed a reduced fertility. We conclude that ZMP buildup in atic-1( $\Delta)$ causes reduced fertility, while the de novo pathway per se has no impact on this phenotype, as ppat-1 $(\Delta)$ is similar to wild-type. Taken together these data show that adsl-1 role in the recycling pathway, and not in the de novo pathway, is essential for germline integrity.

The strong germline defects in ads/-1( $\Delta$ ) prompted us to investigate whether adsl-1 is required during early embryonic development. We therefore examined the viability of ADSL deficient embryos. Given adsl-1( $\Delta$ ) are sterile, we analyzed the progeny of ads/-1( $\Delta) /+$ heterozygotes; if ads/-1 zygotic expression were essential in the embryo one would expect $\sim 25 \%$ embryonic lethality, we observed only $7.7 \%$. Therefore, either adsl-1 is not essential for embryo development or maternal contribution is, at least in part, sufficient. We used RNAi to discriminate between these two hypotheses. Because adsl-1 full penetrant loss-of-function leads to sterility, we performed adsl-1(RNAi) starting at the L4 stage resulting in a hypomorphic phenotype. Under these conditions we observed $32.2 \%$ embryonic lethality in adsl-1(RNAi) (Supplementary Figure 3C), hence maternal expression ensures ads/-1 function during embryonic development. To test whether the de novo pathway contributed to embryonic lethality we performed adsl-1(RNAi) in the ppat-1 $(\Delta)$, resulting in $31.5 \%$ embryonic lethality, similarly to ads/-1(RNAi) alone. The de novo pathway mutants, on the other hand, display normal embryonic development, just as in WT controls: nearly $100 \%$ of the laid eggs hatched in all the de novo pathway mutants, 
bioRxiv preprint doi: https://doi.org/10.1101/277640; this version posted April 6, 2018. The copyright holder for this preprint (which was not certified by peer review) is the author/funder, who has granted bioRxiv a license to display the preprint in perpetuity. It is made available under aCC-BY-NC-ND 4.0 International license.

showing neither ppat-1 nor atic-1 are essential for embryo development. Thus adsl-1 function in the recycling pathway is required for proper embryonic development.

- adsl-1 is required for body size.

The overall morphology observed in adsl-1( $\Delta)$ and ppat-1( $\Delta) ; \operatorname{adsl-1(\Delta )}$ (morphologically indistinguishable from adsl-1( $\Delta)$ single mutants) suggests that cell lineage specification and morphogenesis are not affected, given somatic tissues are properly formed. No defects are observed in the pharynx, intestine or hypodermis (other than the vulva) of adsl-1( $\Delta)$ and animals are viable and mobile. Hence the smaller size is not the result of defects in fate specification. To better understand this phenotype, we measured body length in adult worms. adsl-1( $\Delta$ ) are significantly smaller than wild-type, while the de novo mutants are indistinguishable (Figure $2 \mathrm{C}$ ). ppat-1( $\Delta$ ); adsl-1( $\Delta$ ) are similar to

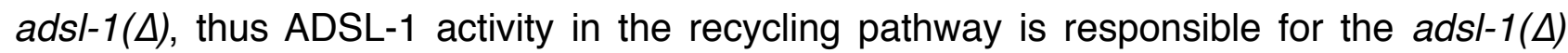
body size phenotype. Given the volume occupied by the germline in adult $C$. elegans we wondered whether sterility could account for the small size in adsl-1( $\Delta$ ), we therefore compared adsl-1( $\Delta)$ to glp-1(e2141) mutants, known to lack a germline when cultured at $25^{\circ} \mathrm{C}$ [22]. Given adult glp-1(e2141) length is in the wild-type range (Figure 2C), sterility cannot account for the small size of adsl-1( $\Delta)$. Noteworthy, newly hatched adsl-1( $\Delta)$ animals are the same size as wild-type and morphologically indistinguishable (not shown), hence size defects manifest during post-embryonic development. We conclude adsl-1 is required, through the recycling pathway, for adult body size independently of the sterility phenotype, presumably by affecting cell size (see discussion).

- adsl-1 is required for developmental timing, but not lifespan.

The small size of adsl-1( $\Delta)$ prompted us to investigate whether post-embryonic development was impaired. Given newly hatched adsl-1( $\Delta)$ are indistinguishable from wildtype, we hypothesized the growth defects were linked to abnormal larval development. Upon hatching and before adulthood, $C$. elegans goes through four larval stages each followed by an inactive state known as lethargus during which a molt takes place [23]. Importantly, this sequence follows a highly reproducible timing under standard culture conditions [24]. We therefore measured timing of post-embryonic development, in purine mutants, using the method described by Olmedo et al [25]. Briefly, newly hatched L1 larvae carrying a transgenic luciferase are cultured in presence of luciferin substrate, emitting luminescence when active [26]. Since larvae cease feeding during lethargus, they stop taking the substrate and a drastic drop in luminescence is observed. Hence, monitoring the luminescence of individual larvae allows to precisely time the occurrence of each larval (high luminescence) and lethargus (low luminescence) phases (Supplementary Figure 4A; [25]). We observed that both adsl-1( $\Delta)$ and ppat-1( $\Delta)$; adsl-1( $\Delta$ ) present a strongly delayed post-embryonic development (Figure 3A, Supplemental Figure 4B). Although all larval and lethargus stages are affected in ads/-1( $\Delta$ ) mutants, the delay is more pronounced as post-embryonic development progresses. By contrast, the overall timing of post-embryonic development is not affected in de novo pathway mutants. We therefore conclude that adsl-1 function in the recycling pathway is required for normal post-embryonic development.

Post-embryonic development, metabolism and the germline are all known to impact longevity in $C$. elegans (reviewed in [27]). Because of ads/-1( $\Delta$ ) sterility and developmental delay we measured the lifespan of the purine mutants. adsl-1( $\Delta)$ mutant has a shorter 
lifespan that wild-type (Figure 3B). By contrast, the de novo pathway mutants' lifespan is in the wild-type range (Figure 3B). Importantly, ppat-1( $\Delta$ ) suppresses the adsl-1( $\Delta$ ) lifespan phenotype as ppat-1( $\Delta)$; adsl-1( $\Delta)$ double mutant survival profile is in the wild-type range (Figure 3B). These results strongly suggest that SZMP buildup in adsl-1( $\Delta$ ), rather than ADSL activity in the recycling pathway, is responsible for the reduced lifespan. Moreover, we conclude that the developmental delay and sterility of adsl-1( $\Delta)$ are not linked to lifespan.

- Imbalance in purine metabolism impacts locomotion

Given the severe muscular and neuronal symptoms of ADSL deficiency patients [9, $11,28]$, we sought to investigate muscle and neural defects in ads/-1( $\Delta$ ) worms. In order to address muscle function, we performed a locomotion assay (Figure 4A; [29]), and observed that ads/-1( $\Delta$ ) mutants were slower than WT (Figure 4B). atic-1( $\Delta$ ) mutants, were also slower than wild-type, albeit to a lesser extent (Figure 4B), suggesting the de novo pathway could have a role in locomotion. However, ppat-1( $\Delta)$ animals were indistinguishable from wild-type, thus de novo pathway per se does not affect locomotion. Moreover, the locomotion defect in adsl-1( $\Delta)$ was not suppressed by ppat-1( $\Delta)$, ppat-1( $\Delta)$; adsl-1( $\Delta)$ was actually slower than adsl-1( $\Delta)$ single mutant. Thus, ADSL activity in the recycling pathway, rather than SZMP buildup, accounts for the adsl-1( $\Delta)$ locomotion defect. By contrast, atic-1( $\Delta)$ locomotion defect was suppressed by ppat-1( $\Delta)$ as ppat-1( $\Delta)$; atic-1 $(\Delta)$ speed was in the wild-type range. Hence, slower locomotion in atic-1 $(\Delta)$ is linked to ZMP build up, rather than the de novo pathway per se.

- AICAR treatment

The ADSL product ZMP is known to activate AMPK by mimicking AMP [6], and AICAR treatment is known to impact muscle function in mice, presumably through ZMP induced AMPK activation [30]. To test whether ZMP is responsible for the locomotion defects in atic-1 $(\Delta)$, we tested whether providing the worms with external AICAR would impact locomotion. We treated late L4 stage wild-type and de novo pathway mutants with AICAR, for 24 hours. Somewhat surprisingly AICAR treatment did not significantly affect locomotion, neither among the genotypes tested, nor comparing with untreated control (Supplementary Figure 5A). Given this surprising result, we performed metabolic profile analysis of AICAR treated worms. As expected, we observed higher levels of AICAR upon treatment, and noticeably a significant buildup of both ZMP and ZTP in all treated genotypes (Supplementary Figure 5B), showing AICAR was efficiently internalized and phosphorylated. As expected ZMP levels are higher in atic-1( $\Delta$ ) than in other genotypes due to cumulative buildup of ZMP from the de novo pathway and from AICAR treatment (Supplementay Figure 5C). Therefore buildup of ZMP in adult worms per se does not explain the reduced locomotion observed atic-1( $\Delta)$. Importantly, while AICAR treatment did not significantly affect ATP levels across genotypes, it lead to an increase in uric acid, particularly in atic-1( $\Delta)$ and ppat-1( $\Delta)$; atic-1( $\Delta)$. Moreover, we also observed, in all treated genotypes, an accumulation of ADSL substrate SZMP and its riboside form SAICAR. Therefore, AICAR treatment leads to increased levels of ZMP as expected, but also of uric acid, ZTP and SZMP (Supplementary Figure 5B). The SAICAR and SZMP accumulation indicates that not only SZMP to ZMP conversion by ADSL is inhibited by AICAR treatment, this reaction can even be reversed, given SZMP was detected in ppat-1( $\Delta)$ and ppat-1 $(\Delta)$; atic-1 $(\Delta)$ mutants (Supplementary Figure 5B, see Supplementary Figure 1B). Accordingly, 
in yeast ADSL enzyme has been shown to convert ZMP in SZMP when ZMP is in excess [31], which seems to also be the case for $C$. elegans ADSL.

- Muscle structure, but not the neuromuscular junction, is disrupted in adsl-1( $\Delta)$

One possible explanation for the locomotion defects observed in atic-1( $\Delta)$ and $\operatorname{adsl-1}(\Delta)$ is that the neuromuscular junction is compromised. We therefore tested whether purine mutants were sensitive to Levamisole and Aldicarb. Both drugs activate acetylcholine receptors at the neuromuscular junction in $C$. elegans, at either the presynapse (Aldicarb) or post-synapse (Levamisole) leading to paralysis of wild-type worms [32], while mutants with defective cholinergic neuromuscular junction are not affected [33, 34]. We found no significant differences among purine mutants and wild-type (Supplementary Figure 6A and B), demonstrating that the neuromuscular junction remains functional upon purine metabolism deficiencies. We therefore tested whether other neuronal functions might be impaired in purine mutants. We performed a standard touch assay as described in the literature [29]. We observed a similar response to touch in all purine mutants as in the wild-type, thus in our experiments the mechanosensory function as well as the behavioral response are intact in purine mutants (Supplementary Figure $6 \mathrm{C})$. In summary we found no evidence for neuronal defects in the purine metabolism mutants tested.

Given these results, we hypothesized that the locomotion defects we observed could be explained by muscle malfunction. We performed an analysis of muscle cell structure through immunohistochemistry and confocal microscopy. As expected, we observed regular parallel arrangement of myosin fibers in the wild-type (Figure 5A), while in adsl-1 $(\Delta)$ mutants $(n=13)$, muscle myosin fibers formed an irregular meshwork and muscle mass seemed reduced (Figure $5 B)$. In atic-1 $(\Delta)(n=14)$, by contrast, the structure of muscle myosin fibers was indistinguishable from wild-type controls (Figure $5 C, n=10$ ), as are ppat-1 $(\Delta)$ (Figure 5D, $\mathrm{n}=13$ ). Furthermore ppat-1 $(\Delta)$; adsl-1( $\Delta)$ (Figure $5 \mathrm{E}, \mathrm{n}=10$ ) displayed the same muscle fiber defects as adsl-1( $\Delta)$, and ppat-1( $\Delta)$; atic-1( $\Delta)$ were indistinguishable from ppat-1( $\Delta$ ) and atic-1( $\Delta$ ) single mutants (Figure $5 \mathrm{~F}, \mathrm{n}=15$ ), showing that de novo pathway deficiency does not affect muscle structure (Figure 5 ). These results indicate that $A D S L$ role in the recycling pathway is required for muscle integrity, likely underlying the locomotion defects we observed.

\section{Discussion}

- A nematode model to study ADSL deficiency and more broadly purine metabolism

We describe here the phenotypes and metabolic profiles associated with ADSL deficiency in the nematode $C$. elegans. This study provides a reference to future studies investigating the underlying molecular mechanisms of the phenotypes observed. Although phenotypes are to some extent genetically separable, we show the most severe ones are linked to ADSL activity in the recycling pathway (Figure 6). This points to the conversion of S-AMP in AMP as the critical enzymatic step in ADSL deficiency. For instance, there is a long standing debate concerning the etiology of the pathology associated with ADSL deficiency. It has been proposed that the SAICAR/S-Ado ratio correlated with specific symptoms [14, 28], while more recent studies found no evidence for such correlation [10, 12]. Studies in human subjects are of limited reach to solve these types of questions, while a genetically amenable model organism shall allow to investigate molecular mechanisms 
underlying specific phenotypes, and potentially provide useful information to the understanding of the disease. $C$. elegans as metazoan model organism provides the means to investigate the metabolic regulation of developmental progression, muscle defects or cell proliferation in the context of ADSL deficiency, as well as in other deficiencies in the purine biosynthesis pathway.

\section{- Purine recycling and GSCs maintenance}

One striking phenotype of $\operatorname{adsl-1}(\Delta)$ is the severe reduction in GSCs, leading to sterility. In adult $C$. elegans the germline is the sole proliferating tissue. More precisely, GSCs in the distal gonad proliferate under the control of Notch signaling from neighboring somatic cells, while in the proximal region the germline nuclei enter meiotic differentiation (reviewed in [35, 36]. Interestingly, deficiencies in both the pyrimidine [19]) and pyridine nucleotides (a.k.a. NAD+) recycling pathways [37] have also been linked to germline defects in $C$. elegans, suggesting nucleotide metabolism to play a critical role in maintaining the GSCs pool. Importantly however, adsl-1( $\Delta)$ phenotype is more severe than in either case. In pnc-1(-) mutants, affecting the pyridines salvage pathway, animals are fertile despite germline defects. In cdd-1/2(-) mutants, deficient for the Cytidine Deaminase in the pyrimidine recycling pathway, GSCs proliferation could be restored with pyrimidine supplementation. By contrast, purine supplementation had no effect on ads/-1( $\Delta)$ GSCs. While one cannot exclude the defects in these mutants are unrelated, the three metabolic pathways are closely connected, it is therefore plausible that germline defects in adsl-1(-), pnc-1(-) and cdd-1/2(-) share a common mechanism, through crosstalk among these metabolic pathways. In that case, the severity of the adsl-1( $\Delta$ ) phenotype remains to be explained. One tempting explanation is that the primary metabolic defect lies on the purine pathway, and that imbalance in the other nucleotide biosynthesis pathways impact purine metabolism as an indirect consequence. It is known that GSCs proliferation is influenced by environmental factors, particularly nutrient availability, and is likely regulated by metabolic signals (reviewed in [38]). Our results are consistent with the purine recycling pathway serving as sensor integrating nucleotide pathways' status, and providing a metabolic signal regulating GSCs proliferation.

Interestingly, the purine biosynthesis pathway has been shown to play a role in the balance between proliferation and differentiation in both stem cells and cancer cells, with inhibition of purine synthesis leading to increased differentiation at the expense of proliferation [39, 40]. A similar mechanism could explain adsl-1( $\Delta$ ) GSCs defect, in that scenario the ADSL deficiency would lead to germline nuclei entering meiosis at the expense of proliferation.

\section{- Purine metabolism and development}

Metazoans often control their development in response to environmental conditions, namely nutriment availability. This control involves metabolic regulation, for instance through Insulin/Insulin-like or mTOR pathways (reviewed in [41]). In $C$. elegans the adult worm size varies depending on the bacterial food source [42], suggesting size is one of the developmental phenotypes subjected to metabolic regulation. This regulation could, in theory be due to metabolic regulation of cell fate decisions, or alternatively be a consequence of metabolic regulation of cell size. In adsl-1( $\Delta)$, based on our analysis, only GSCs and vulval cells present cell fate abnormalities, which have no impact in overall 
adult size. Hence the small size we observe is most likely due to an effect of purine metabolism on cell size.

In addition to size, adsl-1( $\Delta)$ also displays a strong delay during post-embryonic development. In $C$. elegans progression through larval stage and molting cycles is highly regulated, involving oscillations of LIN-42, the orthologue the circadian protein PERIOD [43, 44], reviewed in [45]. The developmental changes associated with this progression involve a tight coordination of different cell lineages across the worm's body, and are very well characterized, as are the genetic pathways involved (reviewed in [46]). By contrast, the upstream mechanisms and signals triggering or modulating these developmental transitions are poorly understood. The quality and amount of food available certainly play a role, as they have been shown to impact the timing of post-embryonic development [21, 47]. The dramatic developmental delay in adsl-1( $\Delta$ ) points at a metabolic regulation of these developmental transitions involving the purine recycling pathway.

One possibility could be that purine synthesis deficiency in adsl-1( $\Delta)$ is limiting ATP levels, causing slow development and smaller size. Moreover, we have observed that in adsl-1( $\Delta$ ) food uptake is reduced compared to wild-type [48], thus not only ATP but limiting overall bulk nutrients could account for the phenotypes observed. Although limiting nutriments and ATP levels are an important factor, and certainly have a sizable impact, several elements suggest this hypothesis does not suffice to explain the adsl-1( $\Delta)$ defects. In eat mutants, known to have feeding impairment, food uptake defects don't always correlate with developmental delay (M.O. and M.A.S. unpublished results). Moreover, in $C$. elegans unfavorable environmental conditions, namely food shortage, induce an alternative larval stage known as dauer [23]. If food intake is limiting in adsl-1( $\Delta)$, it is not so low as to mimic the nutrient scarcity causing dauer formation. Our observation that ads/-1( $\Delta$ ) progresses through post-embryonic development without entering the dauer stage is consistent with bulk nutriments not being the limiting factor. As to ATP levels, our metabolic profiles show them to be relatively high in adsl-1(RNAi), only slightly reduced compared to wild-type. This is not so surprising given adenine or adenosine are available from the bacterial food source could be used to produce ATP despite ADSL deficiency (see Figure 1A). An alternative hypothesis is that post-embryonic developmental and adult size are regulated by the wiring of the purine recycling pathway, rather than the synthesis of its final products. In this view, purine intermediate metabolites could function as metabolic sensors modulating developmental progression.

\section{- Purine metabolism and neuro-muscular function}

Both neuronal and muscle tissues are severely affected in purine metabolism syndromes, suggesting these two tissues are particularly susceptible upon purine metabolism impairment in patients. In our nematode model, we observed locomotion defects associated with both ATIC and ADSL deficiencies. Somewhat surprisingly, no discernible neuronal damage was observed in any purine mutant. All of the genotypes tested displayed sensitivity to Levamisole and Aldicard, similarly to wild-type controls, showing neuromuscular junction was still functional. Moreover, mechanosensory response wasn't affected either in purine mutants. Hence, we could not identify neuronal dysfunctions to account for the locomotion defects.

We did however find evidence for muscle abnormalities that could explain the locomotion defects. Noteworthy, the locomotion defects we observed in atic-1( $\Delta)$ and adsl-1( $\Delta)$ were linked to different mechanisms. Contrary to adsl-1( $\Delta)$, the locomotion delay 
in atic-1 $(\Delta)$ was suppressed in the ppat-1( $\Delta)$; atic-1 $(\Delta)$ double mutant, hence linked to ZMP buildup. One possible explanation is that ZMP accumulation leads to metabolic changes that in turn impact muscle function, possibly through AMPK activation [6]. Importantly, the structure of muscle myosin fibers in atic-1 $(\Delta)$ are undistinguishable from the wild-type, thus muscle integrity seems not to be affected in atic-1( $\Delta)$. The ZMP effect on locomotion, however, is not straightforward; upon AICAR treatment of L4 larvae / young adults ZMP builds up as expected, nonetheless locomotion is not affected. Although the mechanism remains elusive, our results suggest that either ZMP effect on locomotion is developmental and occurs prior to the L4 stage, or ZMP accumulation from external AICAR does not affect the same tissues as endogenous ZMP build up in atic-1( $\Delta)$.

On a side note, because ZMP is an AMPK agonist [6], its riboside form AICAR is often added to culture media in different model organisms including C. elegans (e.g. [49, 50]), to trigger AMPK activation upon intracellular conversion to ZMP. Our results show that increase of ZMP is not the only metabolic consequence of AICAR treatment, therefore caution is recommended when attributing phenotypes to AMPK activation.

As to the locomotion defects in adsl-1( $\Delta)$, our results point to ADSL activity in the purine recycling pathway, given ppat-1( $\Delta)$ does not suppress the locomotion phenotype, quite to the contrary. Furthermore, adsl-1( $\Delta)$ is associated with significant damage in the structure of muscle myosin fibers, suggesting the locomotion defects are caused by muscle defects. While the underlying cause for these defects is yet to be determined, we hypothesize that the developmental delay in adsl-1( $\Delta)$ is linked to muscle defects.

\section{- Purine metabolic network in a metazoan model}

The results we present show the functional conservation of both the de novo and recycling purine biosynthesis pathways in $C$. elegans. Furthermore, we characterized phenotypes associated with the deficiencies in the critical enzymes ADSL and ATIC. While several phenotypes are genetically separable (Figure 6), it stands out that the most severe phenotypes - lack of GSCs, developmental delay and muscle integrity - are associated with ADSL activity in the recycling pathway, whilst deficiencies in the de novo pathway are viable and lead to rather subtle phenotypes - as reduced fertility and slower locomotion. Noticeably, the deletion of ppat-1 - which we show is required for the de novo pathway produces no detectable phenotype in our culture conditions. The phenotypes observed in atic-1 $(\Delta)$, locomotion defects and reduced fertility, are associated with ZMP accumulation, rather than to shortage in purine synthesis through the de novo pathway, as though the de novo pathway per se is dispensable. It might seem paradoxical that the purine de novo pathway is conserved while having no essential function. Indeed a number of biosynthesis pathways, namely amino-acids, have been lost during metazoan evolution [15] likely because amino-acids are available from the food source, as are nucleotides. However, these may not reflect the natural conditions in which $C$. elegans evolved; it is plausible that the de novo pathway is essential in different environments where purines are scarce. Actually, if one draws a parallel with yeast, it's the severity of the adsl-1( $\Delta$ ) phenotypes that seems surprising, considering Ade13 null mutants in $S$. cerevisiae are viable when cultured with adenine [51]. Given purines are available from the food source, one would have expected the worm to synthesize AMP and GMP despite ADSL deficiency. Indeed, external adenine and adenosine can be converted in AMP by Adenine phosphoribosyltransferase and Adenosine kinase respectively, both of which have orthologues in C. elegans (Supplementary Figure 1A), and AMP can be converted in GMP 
(Figure 1A). The severity of adsl-1( $\Delta$ ) phenotypes, suggests that ADSL became essential even when external purines are available. Importantly, in humans, purines are also available from the food source, and all but one diseases affecting purine metabolism are linked to enzymes in the recycling pathway; with the notable exception being AICAribosiduria - deficiency in ATIC, in which ZMP buildup may be a crucial disease factor [2, 3, 13]. The emerging picture is that in metazoans the less energetically costly recycling pathway evolved to become the predominant form of purine metabolism, at least in favorable environmental conditions. Hence, specific functions evolved to strongly rely on the recycling pathway, independently of the abundance of external purine sources. The defects associated with ADSL deficiency, as well as other recycling pathway deficiencies, could be the consequence of unbalanced wiring of the pathway, such as deregulation caused by intermediate metabolites, as much as insufficient synthesis of the final products AMP and GMP.

\section{Materials and Methods}

- Caenorhabditis elegans strains and culture

Nematodes were maintained under standard culture conditions [52, 53], on nematode growth medium (NGM), with Escherichia Coli (strain OP50) as food source, kept at $20^{\circ} \mathrm{C}$ (except strains carrying the transgene ruls32 [pie-1p::GFP::H2B + unc-119(+)], which were kept at $\left.25^{\circ} \mathrm{C}\right)$.

The following Caenorhabditis elegans strains were used in the study: N2 (Bristol, wild-type reference strain) obtained from the Caenorhabditis Genetics Center (CGC), AZ212 ruls32 [pie-1p::GFP::H2B + unc-119(+)] III, CF1903 glp-1(e2141) III, PE254 fels4 [sur-5p::luciferase::GFP + rol-6(su1006)] V, VC2999 R151.2(gk3067) III/hT2 [bli-4(e937) let-?(q782) qls48] (I,III) - parental strain for the hT2 balancer, WBX52 adsl-1(tm3328) I/hT2 [bli-4(e937) let-?(q782) qls48] (I,III), WBX53 adsl-1(tm3328) I; ppat-1(tm6344) III/hT2 [bli-4(e937) let-?(q782) qls48] ppat-1(tm6344) (I,III) (from WBX119 and WBX52), WBX116 ppat-1(tm6344) III, WBX119 ppat-1(tm6344) III, WBX117 atic-1(tm6374) IV, atic-1(tm6374) IV (from WBX119 and WBX117); WBX124 atic-1(tm6374) IV; fels4 [sur-5p::luciferase::GFP + rol-6(su1006)] V (from PE254 and WBX116), WBX127 ppat-1(tm6344) I; adsl-1(tm3328) III/hT2 [bli-4(e937) let-?(q782) qls48] ppat-1(tm6344) (I,III); fels4 [sur-5p::Iuciferase::GFP + rol-6(su1006)] V (from PE254 and WBX53), WBX125 ppat-1(tm6344) III; atic-1(tm6374) IV; fels4 [sur-5p::Iuciferase::GFP + rol-6(su1006)] V (from PE254 and WBX116), WBX130 adsl-1(tm3328) I/hT2 [bli-4(e937) let-?(q782) qls48] (I,III); fels4 [sur-5p::luciferase::GFP + rol-6(su1006)] V (PE254 and WBX53), WBX131 ppat-1(tm6344) III; fels4 [sur-5p::luciferase::GFP + rol-6(su1006)] V (from PE254 and WBX116), WBX147 ppat-1(tm6344) ruls32 [pie-1p::GFP::H2B + unc-119(+)] III (from AZ212 and WBX119), WBX149 adsl-1(tm3328) I; ruls32 [pie-1p::GFP::H2B + unc-119(+)] III/hT2 [bli-4(e937) let-?(q782) qls48] (I,III) (from AZ212 and WBX52) and WBX152 adsl-1(tm3328) I; ppat-1(tm6344) ruls32 [pie-1p::GFP::H2B + unc-119(+)] III/hT2 [bli-4(e937) let-?(q782) qls48] (I,III) (from WBX147 and WBX149). The adsl-1(tm3328), ppat-1(tm6344) and atic-1(tm6374) deletion alleles - referred to as adsl-1 $(\Delta)$, ppat-1 $(\Delta)$ and atic-1 $(\Delta)$ throughout the manuscript - were generated by the lab of Dr. Shohei Mitani, National Bioresource Project, Japan (NBRP), all strains carrying these alleles used in this study were outcrossed a minimum of four times. 
Nematode synchronization: Adult worms were collected with M9 buffer in $15 \mathrm{ml}$ conical tubes, centrifuged $1 \mathrm{~min}$ at $1000 \mathrm{rpm}$ and supernatant was discarded. Worm bleach solution $(\mathrm{NaOH} 1 \mathrm{M}, \mathrm{NaOCl} 0.25 \mathrm{M})$ was added until carcasses dissolve and eggs release ( 8 min). Eggs were washed three times with M9 buffer transferred onto NGM seeded plates.

RNAi: In order to generate the adsl-1(RNAi) feeding vector a PCR fragment was amplified with oligomers 5'-CTTCTTCCGGCAGAATCATCCAGTG-3' and 5'TGACTGTCGGACGTACTCATTACC-3' and inserted onto L4440 plasmid [54]. For RNAi experiments, HT115(DE3) bacteria transformed with either the adsl-1(RNAi) feeding vector, or L4440 (Control), were cultured overnight at $37^{\circ} \mathrm{C}$ in LB $150 \mathrm{mg} / \mathrm{L}$ Ampicillin and induced with 2 mM IPTG 1-2 h prior seeding onto NGM 2 mM IPTG 150 mg/L Ampicillin, in which synchronous populations of worms were cultured from hatching to adulthood.

- Yeast heterologue rescue assay

Yeast expression vectors were constructed by inserting full length cDNAs of ppat-1 ( a m p i ified using oligomers 5 ' CACTAAATTACCGGATCAATTCGGGGGATCCATGTGTGGTATATTTGGGATTG-3' and 5’CATAACTAATTACATGATGCGGCCCTCCTGCAGTTATAAATCGATAGCAACTGG-3'), ads/-1 (5'-CGGGATCCGTATCATGGCCTCCGAAGACAAGT-3' and 5'CCAATGCATGAATCAAACATCCAGCTGAACATTTCC-3') and atic-1b (5'G A A G A C T C G A C G A T T G TGTGAAATGACCGAC-3 ' and 5 ' ATAAGAATGCGGCCGCATCTAATGGTGGAAGAGACGAAGTC-3') onto pCM189 plasmid [55]. ade $4 \Delta$ yeast strain, genotype ade $4:$ KanMX4; his3 $\Delta 1$; leu2 $\Delta 0$; lys $2 \Delta 0$; ura3 $\Delta 0$, was used to test heterologous rescue by ppat-1, ade13 $\Delta$ yeast strain, genotype ade13::kanMX4; his3 $\Delta$; leu2 $\Delta$; ura3 $\Delta$, was used to test heterologous rescue by adsl-1 and ade16 $\Delta$ ade17 double mutant, genotype ade16::KanMX4; ade17::KanMX4; his3 $\Delta$; leu2 $\Delta 0$; ura3 $\Delta 0$; lys $2 \Delta 0$ was used to test heterologous rescue with atic- $1 b$.

\section{- Imaging}

For adult morphology, DIC (Differential Interference Contrast, a.k.a. Nomarski) images were taken with a Leitz Diaplan microscope. Adult worms were mounted in M9 buffer, on $2 \%$ agarose cushion. Images were acquired with VisiCam 5.0 camera, analyzed and processed with Fiji software. For imaging of pie-1p::GFP::H2B germline reporter, synchronized adult worms were mounted in M9 Buffer $5 \mathrm{mM}$ Levamisole onto $2 \%$ agarose cushion. Images were acquired with an Olympus IX81 microscope under an 40X oilimmersion objective, with Hamamatsu Orca R2 camera. Images were analyzed with Fiji software. For size measurements synchronized populations of young adult worms were used, each worm was measured 3 times on three different pictures. Images were acquired on a Nikon SMZ18 stereo microscope with VisiCam 5.0 camera, analyzed and processed with Fiji software.

\section{- Immunostainings}

Immunostaining was performed on synchronized populations of worms, N2 and de novo pathway mutants were fixed $72 \mathrm{~h}$ after synchronization, adsl-1( $\Delta)$ and ppat-1( $\Delta)$; adsl-1( $\Delta)$ were fixed $96 \mathrm{~h}$ after synchronization. Fixation protocol was adapted from Benian et al. [56]. Worms were collected in ice cold PBS buffer on $1.5 \mathrm{ml}$ tubes, centrifuged and supernatant was discarded, and fixation solution was added (made fresh, $1 \mathrm{ml}$ of MRBW 
buffer $\mathrm{KCl} 320$ mM, NaCl 80 mM, EGTA 40 mM, Spermidine 20 mM, Pipes 60 mM was added to $2.8 \mathrm{ml}$ of Methanol and $200 \mu \mathrm{l}$ of Formaldehyde $20 \%$ ), followed by five cycles of freezing in liquid $\mathrm{N} 2$ and thawing and $1 \mathrm{~h}$ incubation at $4{ }^{\circ} \mathrm{C}$ with gentle agitation, fixation solution was replaced by a permeabilization solution (PBS, Triton X-100 2\%, B-mercaptoethanol $1 \%$ ) and incubated $1 \mathrm{~h}$ at room temperature, permeabilization solution was renewed and incubated $1 \mathrm{~h}$ at room temperature, followed by three washes in PBSt (PBS, Triton X-100 0.5\%), after which worms were incubated with primary antibody (diluted 1:1000 in PBSt 5\% Fetal Calf Serum) overnight at $4^{\circ} \mathrm{C}$ with gentle agitation, washed three times in PBSt and incubated with secondary antibody (diluted 1:500 in PBSt 5\%FCS). Monoclonal antibody 5-6 (Developmental Studies Hybridoma Bank) directed against muscle myosin was used as primary antibody, anti-mouse Alexa555 (Molecular Probes) was used as secondary antibody, worms were mounted on Fluoroshield (Sigma). Images were acquired with a Leica DM6000 TCS SP5 confocal microscope using LAS AF (Leica) software.

\section{- Metabolic profiling by HPLC}

For an extraction, 7500 young adult worms, $72 \mathrm{~h}$ after synchronization, were collected with ice cold water, onto $15 \mathrm{ml}$ conical tubes, and centrifuged at $1000 \mathrm{rpm}, 1 \mathrm{~min}$ and $4^{\circ} \mathrm{C}$, supernatant was discarded eliminating most bacteria. The nematode pellet was washed with $10 \mathrm{ml}$ of cold Hepes $10 \mathrm{mM}$ and centrifuged. The worm pellet was resuspended in Hepes $10 \mathrm{mM}, 1 \mathrm{ml}$ of worm suspension is transferred onto $4 \mathrm{ml}$ of absolute ethanol at $80^{\circ} \mathrm{C}$, incubated for $5 \mathrm{~min}$ and cooled on ice for $10 \mathrm{~min}$. Samples were desiccated on a rotovapor apparatus, the dry residue was resuspended in $350 \mu \mathrm{l}$ of HyClone Molecular Biology-Grade Water (GE Healthcare) and centrifuged at $21000 \mathrm{~g}, 5 \mathrm{~min}$ at $4^{\circ} \mathrm{C}$, supernatant was transferred and centrifuged again at $21000 \mathrm{~g}, 1 \mathrm{~h}$ at $4^{\circ} \mathrm{C}$. The last supernatant was used for HPLC. Metabolites were separated by ionic chromatography on an ICS3000 chromatography station (Dionex, Sunnyvale, CA), and measured by an UV diode array (Ultimate 3000 RS, Dionex). Metabolites separation was done on a carbopacPA1 column $(250 \times 2 \mathrm{~mm}$; Dionex $)$ using a sodium acetate gradient [57]. Metabolite levels were normalized relative to dry weight; the same amount of worms used in the extractions were collected in ice cold water, $72 \mathrm{~h}$ after synchronization, centrifuged, washed 5 times with water, allowed to evaporate for 4 days at $70^{\circ} \mathrm{C}$ and weighed.

\section{- Lifespan}

Upon synchronization worms were transferred onto a new plate every $24 \mathrm{~h}$, and scored. Immobile worms unresponsive to touch were considered dead.

\section{- Locomotion and Behavioral assays}

Locomotion speed measurement was adapted from [29]; images were acquired on a Leica Z16 APO Macroscope and processed with Fiji software, worm displacement was measured by tracking the pharynx, one frame per second.

Aldicarb (Sigma) and levamisole (Sigma) assays were performed as described in [34], synchronized adult worms were placed on $1 \mathrm{mM}$ Aldicarb or $0.4 \mathrm{mM}$ Levamisole plates for $2 \mathrm{~h}$, after which plates were tapped 10 times and worm movement was scored.

Mechanosensory response assay was performed as described in [29], synchronized adult worms were isolated on plates without food for $3 \mathrm{~min}$, and stimulated with an eyelash on 
the posterior pharynx, and scored for avoidance behavior, each worm was stimulated 10 time 3 min apart.

- Post embryonic development

Monitoring of post-embryonic development was performed as described by Olmedo et al. [25]. In brief, upon synchronization, 20 embryos per $\mu \mathrm{l}$ in $\mathrm{M} 9$ buffer overnight with agitation, allowing L1 larvae to hatch. A single L1 larvae was added per well in 96-well plates with S-Basal media, $10 \mathrm{~g} / \mathrm{L} \mathrm{E}$. coli OP50 (wet weight) and $100 \mu \mathrm{M}$ D-Luciferin. The luminescence was measured with a Berthold Centro LB960 XS ${ }^{3}$ during 1 second at 5-min intervals, within a temperature controlled Panasonic MIR-154 incubator.

- Statistics

Statistical analysis was done using Graphpad, Excel, Numbers and R softwares. We consider statistical test to be significant when pValue $<0.01$.

\section{Acknowledgements}

Some $C$. elegans strains were provided by the CGC (https://cgc.umn.edu), which is funded by NIH Office of Research Infrastructure Programs (P40 OD010440). We thank Wormbase, which is supported by National Institutes of Health (NIH) grant U41 HG002223. This work was supported by an AFM-Téléthon Trampoline grant (ref. 18313). $\mathrm{RM}$ is supported by AFM-Téléthon PhD scholarship (ref. 20450). RM stay in M. A-S. lab supported by a Short Term Scientific Mission financed by GENiE (COST Action BM1408). M.A-S and M.O are supported by the Ramón y Cajal program of the Spanish Ministerio de Economía y Competitividad, RYC-2010-06167 and RYC-2014-15551, respectively. Confocal microscopy, and locomotion assay imaging were done in the Bordeaux Imaging Center, a service unit of the CNRS-INSERM and Bordeaux University, member of the national infrastructure France Biolmaging. Deletion alleles adsl-1(tm3328), ppat-1(tm6344) and atic-1(tm6374) were generated by the National Bioresource Project, Japan, directed by Shohei Mitani. We thank Yuji Kohara for providing cDNAs. We thank the members of the B. D-F. lab for helpful discussions, and particularly M. Moenner for critically reading the manuscript.

\section{Literature cited}

1. Ljungdahl P.O., and Daignan-Fornier B. (2012). Regulation of amino acid, nucleotide, and phosphate metabolism in Saccharomyces cerevisiae. Genetics. 190, 885-929.

2. Nyhan W.L. (2005). Disorders of purine and pyrimidine metabolism. Mol Genet Metab. 86, 25-33.

3. Kelley R.E., and Andersson H.C. (2014). Disorders of purines and pyrimidines. Handb Clin Neurol. 120, 827-838.

4. Bochner B.R., and Ames B.N. (1982). ZTP (5-amino 4-imidazole carboxamide riboside 5'-triphosphate): a proposed alarmone for 10-formyl-tetrahydrofolate deficiency. Cell. 29, 929-937.

5. Pinson B., Vaur S., Sagot I., Coulpier F., Lemoine S., and Daignan-Fornier B. (2009). Metabolic intermediates selectively stimulate transcription factor interaction and modulate phosphate and purine pathways. Genes Dev. 23, 1399-1407.

6. Jin X., Townley R., and Shapiro L. (2007). Structural insight into AMPK regulation: ADP comes into play. Structure. 15, 1285-1295. 
7. Keller K.E., Tan I.S., and Lee Y.-S. (2012). SAICAR stimulates pyruvate kinase isoform $\mathrm{M} 2$ and promotes cancer cell survival in glucose-limited conditions. Science. 338, 1069-1072.

8. Gooding J.R., Jensen M.V., Dai X., Wenner B.R., Lu D., Arumugam R., Ferdaoussi M., MacDonald P.E., and Newgard C.B. (2015). Adenylosuccinate Is an Insulin Secretagogue Derived from Glucose-Induced Purine Metabolism. Cell Rep. 13, 157-167.

9. Jurecka A., Zikanova M., Kmoch S., and Tylki-Szymańska A. (2015). Adenylosuccinate lyase deficiency. J Inherit Metab Dis. 38, 231-342.

10. Jurecka A., Zikanova M., Tylki-Szymanska A., Krijt J., Bogdanska A., Gradowska W., Mullerova K., Sykut-Cegielska J., Kmoch S., and Pronicka E. (2008). Clinical, biochemical and molecular findings in seven Polish patients with adenylosuccinate lyase deficiency. Mol Genet Metab. 94, 435-442.

11. Spiegel E.K., Colman R.F., and Patterson D. (2006). Adenylosuccinate lyase deficiency. Mol Genet Metab. 89, 19-31.

12. Zikanova M., Skopova V., Hnizda A., Krijt J., and Kmoch S. (2010). Biochemical and structural analysis of 14 mutant ADSL enzyme complexes and correlation to phenotypic heterogeneity of adenylosuccinate lyase deficiency. Hum Mutat. 31, 445-455.

13. Marie S., Heron B., Bitoun P., Timmerman T., Van den Berghe G., and Vincent M.F. (2004). AICA-ribosiduria: a novel, neurologically devastating inborn error of purine biosynthesis caused by mutation of ATIC. Am J Hum Genet. 74, 1276-1281.

14. Race V., Marie S., Vincent M.F., and Van den Berghe G. (2000). Clinical, biochemical and molecular genetic correlations in adenylosuccinate lyase deficiency. Hum Mol Genet. 9, 2159-2165.

15. Payne S.H., and Loomis W.F. (2006). Retention and loss of amino acid biosynthetic pathways based on analysis of whole-genome sequences. Eukaryot Cell. 5, 272-276.

16. Rebora K., Desmoucelles C., Borne F., Pinson B., and Daignan-Fornier B. (2001). Yeast AMP pathway genes respond to adenine through regulated synthesis of a metabolic intermediate. Mol Cell Biol. 21, 7901-7912.

17. Keller K.E., Doctor Z.M., Dwyer Z.W., and Lee Y.-S. (2014). SAICAR induces protein kinase activity of PKM2 that is necessary for sustained proliferative signaling of cancer cells. Mol Cell. 53, 700-709.

18. Praitis V., Casey E., Collar D., and Austin J. (2001). Creation of low-copy integrated transgenic lines in Caenorhabditis elegans. Genetics. 157, 1217-1226.

19. Chi C., Ronai D., Than M.T., Walker C.J., Sewell A.K., and Han M. (2016). Nucleotide levels regulate germline proliferation through modulating GLP-1/Notch signaling in C. elegans. Genes Dev. 30, 307-320.

20. Watson E., MacNeil L.T., Ritter A.D., Yilmaz L.S., Rosebrock A.P., Caudy A.A., and Walhout A.J. (2014). Interspecies systems biology uncovers metabolites affecting $C$. elegans gene expression and life history traits. Cell. 156, 759-770.

21. Houthoofd K., Braeckman B.P., Lenaerts I., Brys K., De Vreese A., Van Eygen S., and Vanfleteren J.R. (2002). Axenic growth up-regulates mass-specific metabolic rate, stress resistance, and extends life span in Caenorhabditis elegans. Exp Gerontol. 37, 1371-1378.

22. Priess J.R., Schnabel H., and Schnabel R. (1987). The glp-1 locus and cellular interactions in early C. elegans embryos. Cell. 51, 601-611.

23. Cassada R.C., and Russell R.L. (1975). The dauerlarva, a post-embryonic developmental variant of the nematode Caenorhabditis elegans. Dev Biol. 46, 326-342.

24. Byerly L., Cassada R.C., and Russell R.L. (1976). The life cycle of the nematode Caenorhabditis elegans. I. Wild-type growth and reproduction. Dev Biol. 51, 23-33. 
25. Olmedo M., Geibel M., Artal-Sanz M., and Merrow M. (2015). A High-Throughput Method for the Analysis of Larval Developmental Phenotypes in Caenorhabditis elegans. Genetics. 201, 443-448.

26. Lagido C., Pettitt J., Flett A., and Glover L.A. (2008). Bridging the phenotypic gap: real-time assessment of mitochondrial function and metabolism of the nematode Caenorhabditis elegans. BMC Physiol. 8, 7.

27. Antebi A. (2013). Steroid regulation of C. elegans diapause, developmental timing, and longevity. Curr Top Dev Biol. 105, 181-212.

28. Jaeken J., Wadman S.K., Duran M., van Sprang F.J., Beemer F.A., Holl R.A., Theunissen P.M., de Cock P., van den Bergh F., and Vincent M.F. (1988). Adenylosuccinase deficiency: an inborn error of purine nucleotide synthesis. Eur $\mathrm{J}$ Pediatr. 148, 126-131.

29. Hart A.C. (2006). Behavior. WormBook.

30. Narkar V.A., Downes M., Yu R.T., Embler E., Wang Y.-X., Banayo E., Mihaylova M.M., Nelson M.C., Zou Y., Juguilon H. et al. (2008). AMPK and PPARd Agonists Are Exercise Mimetics. Cell. 134, 405-415.

31. Rebora K., Laloo B., and Daignan-Fornier B. (2005). Revisiting Purine-Histidine Cross-Pathway Regulation in Saccharomyces cerevisiae: A Central Role for a Small Molecule. Genetics. 171, 61-70.

32. Lewis J.A., Wu C.H., Berg H., and Levine J.H. (1980). The genetics of levamisole resistance in the nematode Caenorhabditis elegans. Genetics. 95, 905-928.

33. Fleming J.T., Squire M.D., Barnes T.M., Tornoe C., Matsuda K., Ahnn J., Fire A., Sulston J.E., Barnard E.A., Sattelle D.B. et al. (1997). Caenorhabditis elegans levamisole resistance genes lev-1, unc-29, and unc-38 encode functional nicotinic acetylcholine receptor subunits. J Neurosci. 17, 5843-5857.

34. Gendrel M., Rapti G., Richmond J.E., and Bessereau J.L. (2009). A secreted complement-control-related protein ensures acetylcholine receptor clustering. Nature. 461, 992-996.

35. Kimble J., and Crittenden S.L. (2005). Germline proliferation and its control. WormBook. 1-14.

36. Hansen D., and Schedl T. (2013). Stem cell proliferation versus meiotic fate decision in Caenorhabditis elegans. Adv Exp Med Biol. 757, 71-99.

37. Vrablik T.L., Huang L., Lange S.E., and Hanna-Rose W. (2009). Nicotinamidase modulation of NAD+ biosynthesis and nicotinamide levels separately affect reproductive development and cell survival in C. elegans. Development. 136, 3637-3646.

38. Hubbard E.J., Korta D.Z., and Dalfó D. (2013). Physiological control of germline development. Adv Exp Med Biol. 757, 101-131.

39. Oburoglu L., Tardito S., Fritz V., de Barros S.C., Merida P., Craveiro M., Mamede J., Cretenet G., Mongellaz C., An X. et al. (2014). Glucose and glutamine metabolism regulate human hematopoietic stem cell lineage specification. Cell Stem Cell. 15, 169-184.

40. Tardito S., Oudin A., Ahmed S.U., Fack F., Keunen O., Zheng L., Miletic H., Sakariassen P.Ø., Weinstock A., Wagner A. et al. (2015). Glutamine synthetase activity fuels nucleotide biosynthesis and supports growth of glutamine-restricted glioblastoma. Nat Cell Biol. 17, 1556-1568.

41. Danielsen E.T., Moeller M.E., and Rewitz K.F. (2013). Nutrient signaling and developmental timing of maturation. Curr Top Dev Biol. 105, 37-67.

42. Samuel B.S., Rowedder H., Braendle C., Félix M.A., and Ruvkun G. (2016). Caenorhabditis elegans responses to bacteria from its natural habitats. Proc Natl Acad Sci U S A. 113, E3941-9. 
43. Monsalve G.C., Van Buskirk C., and Frand A.R. (2011). LIN-42/PERIOD controls cyclical and developmental progression of C. elegans molts. Curr Biol. 21, 2033-2045.

44. Edelman T.L., McCulloch K.A., Barr A., Frøkjær-Jensen C., Jorgensen E.M., and Rougvie A.E. (2016). Analysis of a lin-42/period Null Allele Implicates All Three Isoforms in Regulation of Caenorhabditis elegans Molting and Developmental Timing. G3 (Bethesda). 6, 4077-4086.

45. Monsalve G.C., and Frand A.R. (2012). Toward a unified model of developmental timing: A "molting" approach. Worm. 1, 221-230.

46. Rougvie A.E., and Moss E.G. (2013). Developmental transitions in C. elegans larval stages. Curr Top Dev Biol. 105, 153-180.

47. MacNeil L.T., Watson E., Arda H.E., Zhu L.J., and Walhout A.J. (2013). Diet-induced developmental acceleration independent of TOR and insulin in C. elegans. Cell. 153, 240-252.

48. Rodríguez-Palero M.J., López-Díaz A., Marsac R., Gomes J.E., Olmedo M., and Artal-Sanz M. (2018). An automated method for the analysis of food intake behaviour in Caenorhabditis elegans. Sci Rep. 8, 3633.

49. Ahmad W., and Ebert P.R. (2017). Metformin Attenuates A $\beta$ Pathology Mediated Through Levamisole Sensitive Nicotinic Acetylcholine Receptors in a C. elegans Model of Alzheimer's Disease. Mol Neurobiol. 54, 5427-5439.

50. Moreno-Arriola E., El Hafidi M., Ortega-Cuéllar D., and Carvajal K. (2016). AMPactivated protein kinase regulates oxidative metabolism in Caenorhabditis elegans through the NHR-49 and MDT-15 transcriptional regulators. PLoS One. 11, e0148089.

51. Dorfman B.Z. (1969). The isolation of adenylosuccinate synthetase mutants in yeast by selection for constitutive behavior in pigmented strains. Genetics. 61, 377-389.

52. Brenner S. (1974). The genetics of Caenorhabditis elegans. Genetics. 77, 71-94.

53. Stiernagle T. (2006). Maintenance of C. elegans. WormBook. 1-11.

54. Timmons L., and Fire A. (1998). Specific interference by ingested dsRNA. Nature. 395, 854.

55. Gari E., Piedrafita L., Aldea M., and Herrero E. (1997). A set of vectors with a tetracycline-regulatable promoter system for modulated gene expression in Saccharomyces cerevisiae. Yeast. 13, 837-848.

56. Benian G.M., Tinley T.L., Tang X., and Borodovsky M. (1996). The Caenorhabditis elegans gene unc-89, required fpr muscle M-line assembly, encodes a giant modular protein composed of Ig and signal transduction domains. J Cell Biol. 132, 835-848.

57. Ceballos-Picot I., Le Dantec A., Brassier A., Jaïs J.P., Ledroit M., Cahu J., Ea H.K., Daignan-Fornier B., and Pinson B. (2015). New biomarkers for early diagnosis of Lesch-Nyhan disease revealed by metabolic analysis on a large cohort of patients. Orphanet J Rare Dis. 10, 7.

\section{Figure Legends}

Figure 1 - Purine metabolism in C. elegans. (A) Schematics of the Purine Biosynthesis pathways in $C$. elegans based on sequence homology. Enzymes subjected to functional analysis are represented in red, other enzymes in green and metabolites in black. (B) Drop test to assess adenine auxotrophy in yeast $S$. cerevisiae mutants deficient for enzymes

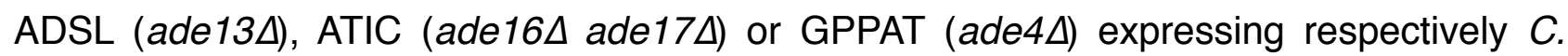
elegans genes adsl-1, atic-1b and ppat-1. Expression of $S$. cerevisiae genes $A D E 13$, $A D E 17$ and $A D E 4$ was used as positive control, and expression vector without insert as 
negative control. A culture with adenine is shown as auxotrophy control. In all drop tests presented, four drops are shown per condition, corresponding to serial dilutions (1:10) of cellular suspensions, with deceasing cell concentrations from left to right. (C) Zoom in on HPLC chromatogram peaks of specific metabolites SAICAR (SZMP riboside form), SZMP, sAdo (succinyl-Adenosine, SAMP riboside form), SAMP, ATP and GTP, upon adsl-1(RNAi) and ppat-1(D); adsl-1(RNAi). (D) Zoom in on HPLC chromatogram peaks of specific metabolites SAICAR (SZMP riboside form), SZMP, AICAR (ZMP riboside form), ZMP, ATP and GTP, in atic-1( $\Delta)$ and ppat-1( $\Delta)$; atic-1( $\Delta)$. Abreviations: Ade - adenine, Ado adenonsine, SAdo - succinyl-adenosine, Ino - Inosine, Hypox - hypoxantine, Xant Xantine, Gua - guanine, Guo - guanosine. In panels (C) and (D), scales are adjusted differently among metabolites, in order to highlight differences between genotypes.

Figure 2 - Developmental defects in purine mutants. (A) DIC whole body images of representative young adults for each of the genotypes studied (bar= $200 \mu \mathrm{m}$ ). (B) Fluorescence micrographs of worms expressing $\mathrm{H} 2 \mathrm{~B}:: \mathrm{GFP}$ in the germline under the control of the pie-1 promoter. Arrows point at rare germ nuclei expressing H2B::GFP; spotty pattern throughout adsl-1( $\Delta)$ and ppat-1( $\Delta)$; adsl-1( $\Delta)$ corresponds to intestinal autofluorescence (bar= $25 \mu \mathrm{m}$ ). (C) Tukey box plot depicting body length of all purine mutants and glp-1(-) for comparison ( ${ }^{*}$ - statistically significant difference relative to wild-type - one way ANOVA; $n=30$ for all genotypes).

Figure 3 - Life traits in purine mutants. (A) - Graph depicting duration of post-embryonic developmental stages, white bars represent larval stages, from L1 to L4, orange bars represent lethargus phases consecutive to each larval stage ${ }^{*}$ - statistically significant difference relative to wild-type - one way ANOVA; see detailed representation of the data in Supplementary Figure 4). (B) Graph depicting fraction of alive worms over time for all genotypes studied $\left(^{*}\right.$ - statistically significant difference relative to wild-type - KaplanMeier; total combined of three independent experiments for each genotype, wild-type $\mathrm{n}=$ 106, ppat-1 $(\Delta) \mathrm{n}=91$, atic $-1(\Delta) \mathrm{n}=126$, ppat $-1(\Delta) ;$ atic-1 $(\Delta) \mathrm{n}=149$, ads $-1(\Delta) \mathrm{n}=227$, ppat-1 $(\Delta)$; adsl-1 $(\Delta) \mathrm{n}=113)$.

Figure 4 - Locomotion of purine mutants. (A) Cartoon schematics of the locomotion behaviour assay. (B) Graph depicting the average locomotion speed in all purine mutants (error bar - standard error of the mean; $n=30$ in all genotypes except wild-type and ppat-1 $(\Delta)$; atic-1 $(\Delta) \mathrm{n}=40 ;$ * - statistically significant difference relative to wild-type Student's t-test).

Figure 5 - Muscle structure in purine mutants. Confocal micrographs of muscle myosin immunostaining in young adult's body wall muscle cells of all studied purine mutants (bar= $10 \mu \mathrm{m})$. (A) Wild-type. (B) adsl-1( $\Delta)$. (C) atic-1( $\Delta)$. (D) ppat-1( $\Delta)$. (E) ppat-1( $\Delta)$; adsl-1( $\Delta)$. (D) ppat-1( $\Delta)$; atic-1( $\Delta)$.

Figure 6 - Summary of phenotypes linked to deficiencies in the purine biosynthesis pathway in $C$. elegans reported in this study.

Supplementary Figure 1 - Purine biosynthesis pathway conservation in C. elegans. (A) Schematics of the Purine Biosynthesis pathways in $C$. elegans based on sequence 
homology, and table of comparison with both human and yeast ( $S$. cerevisiae) orthologues. Enzymes subject to functional analysis are in red. (B) Schematics of predicted purine biosynthesis pathways in adsl-1( $\Delta)$, atic-1 $(\Delta)$ and ppat-1( $\Delta)$ mutants; large font represents intermediate metabolite accumulation. (C) Representative example of a chromatogram, absorbance at $260 \mathrm{~nm}$, of wild-type worms, in mock (control) RNAi condition.

Supplementary Figure 2 - Sequence alignment of $C$. elegans PPAT-1, ADSL-1 and ATIC-1 with closest orthologue in humans, rat (Rattus norvegicus), fly (Drosophila melanogaster) and yeast $(S$. cerevisiae). Grey boxes indicate similar residues, black boxes indicate identical residues.

Supplementary Figure 3 - Developmental defects in purine mutants. (A) Representative DIC micrographs of the vulva region in young adults of wild-type, ppat-1( $\Delta)$, adsl-1( $\Delta)$ and ppat-1 $(\Delta)$; adsl-1 $(\Delta)$ genotypes (bar=20 $\mu \mathrm{m}$ ). (B) Graph depicting the average number of eggs laid by the de novo pathway mutants, and AICAR treated ppat-1( $\Delta)$; atic-1( $\Delta)(0.1$ $\mathrm{mM}$ ) and wild-type $(0.5 \mathrm{mM})$ worms (error bar - standard error of the mean; $\mathrm{n}=30$ in all samples; * - statistically significant difference relative to wild-type - Student's t-test). (C) Graph displaying the percentage of embryonic lethality (unhatched eggs) upon adsl-1(RNAi) and mock (control) RNAi in both wild-type and ppat-1( $\Delta$ ).

Supplementary Figure 4 - (A) Representative bioluminescence profiles of a single wildtype (left, blue line) and adsl-1( $\Delta$ ) mutant (right, orange line) individuals over time. Arrows indicate drop in luminescence observed during lethargus phase. (B) Detailed representation of the primary data presented in figure $3 \mathrm{~A}$, each circle corresponds to an individual observation, bars indicate median, 2nd and 3rd quartiles. L1 through L4 refer to the four larval stages and M1 though M4 refer to the lethargus phases following each larval stage. For better visualization adsl-1( $\Delta)$ and ppat-1( $\Delta)$; adsl-1( $\Delta)$ in L3, L4, M3 and M4 are represented on a different time scale. ( ${ }^{*}$ - statistically significant difference compared to wild-type; one-way ANOVA).

Supplementary Figure 5 - AICAR treatment and locomotion. (A) Graph depicting the average locomotion speed in de novo pathway mutants treated with AICAR (error bar standard error of the mean; $\mathrm{n}=30$ in ppat-1( $\Delta)$ and atic-1 $(\Delta), \mathrm{n}=40$ in wild-type and ppat-1( $\Delta)$; atic-1 $(\Delta)$; * - statistically significant difference relative to wild-type - Student's ttest). (B) Zoom in on HPLC chromatogram peaks of specific metabolites AICAR, ZMP, SAICAR, SZMP, ZTP, uric acid, ATP and GTP, upon $0.5 \mathrm{mM}$ AICAR treatment. (C) Zoom in on HPLC chromatogram peaks of ZMP upon $0.5 \mathrm{mM}$ AICAR treatment, with the four genotypes analyzed represented on the sale scale.

Supplementary Figure 6 - Neuromuscular defects in purine mutants. (A) Percentage of paralysed worms upon Aldicarb treatment, in all studied genotypes. (B) Percentage of paralysed worms upon Levamisole treatment, in all studied genotypes. (C) Graph presenting the percentage of observed responses to mechanosensorial stimulation in all studied genotypes. 
A

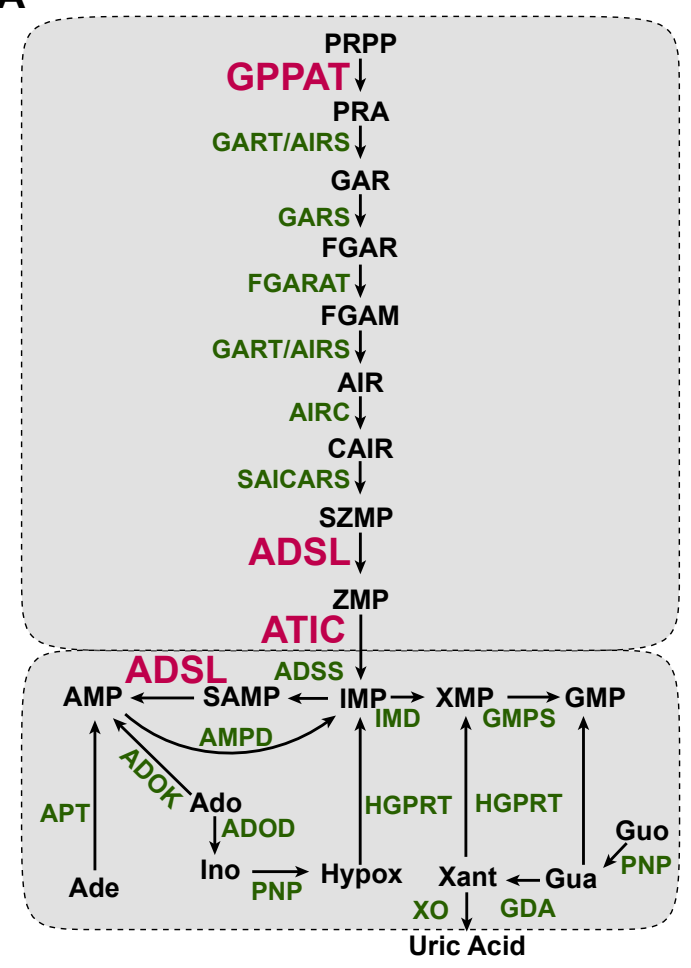

B

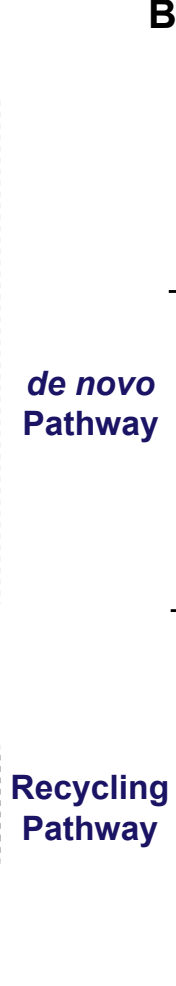

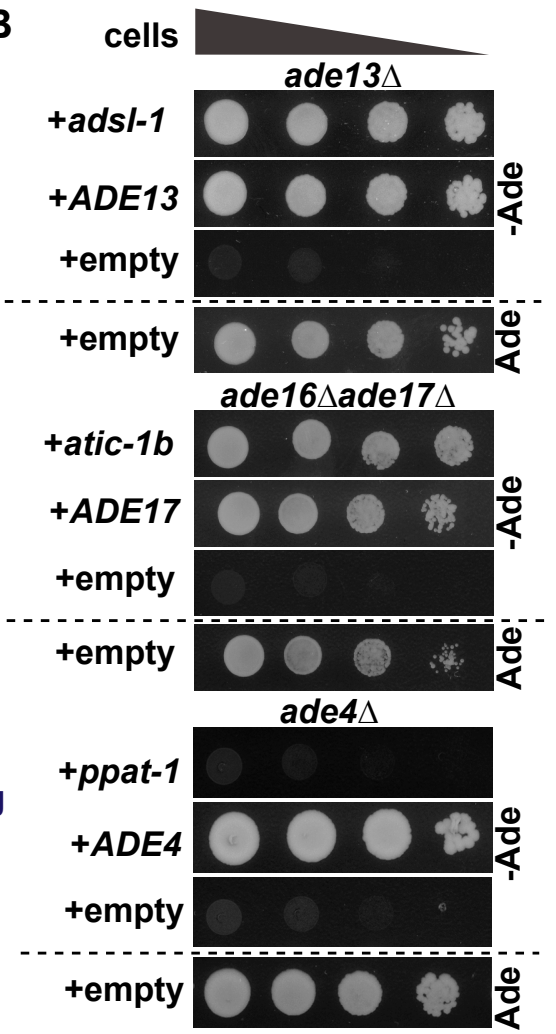

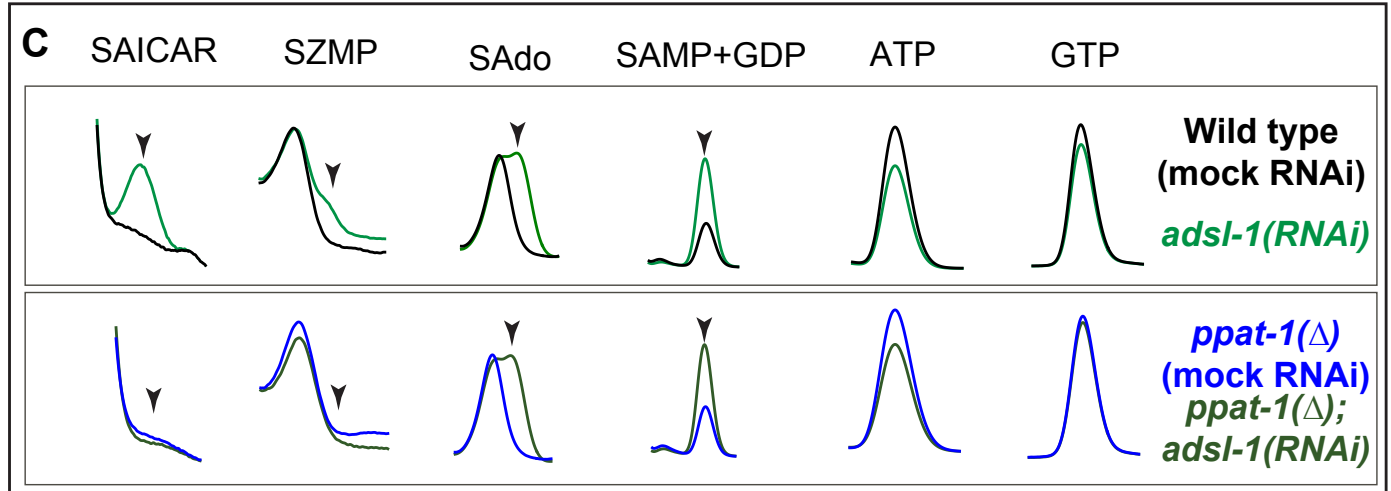

\begin{tabular}{|c|c|c|c|c|c|c|}
\hline SAICAR & SZMP & AICAR & ZMP & ATP & GTP & \\
\hline & & & $\Lambda$ & & & $\begin{array}{l}\text { Wild-type } \\
\text { atic-1 }(\Delta)\end{array}$ \\
\hline & Ar & & & $A$ & $A$ & $\begin{array}{c}\text { ppat-1( }(\Delta) \\
\text { ppat-1 }(\Delta) ; \\
\text { atic-1 }(\Delta)\end{array}$ \\
\hline
\end{tabular}

Figure 1 


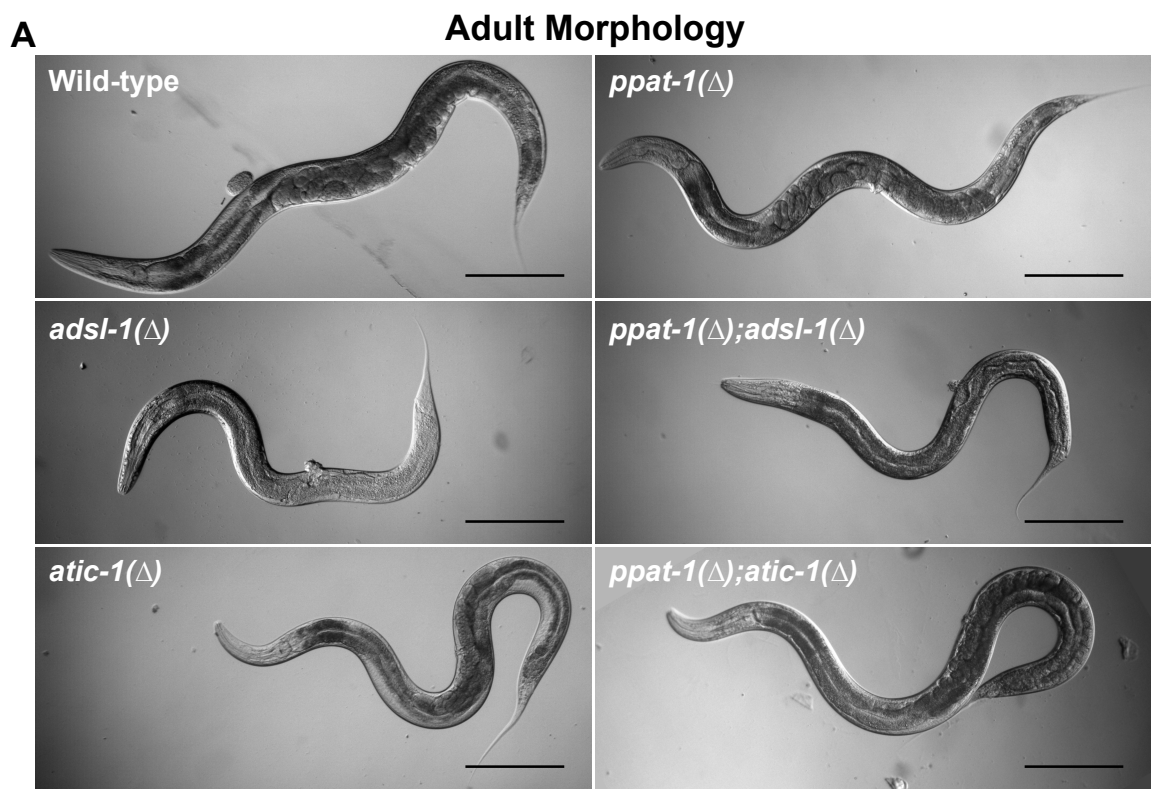

B

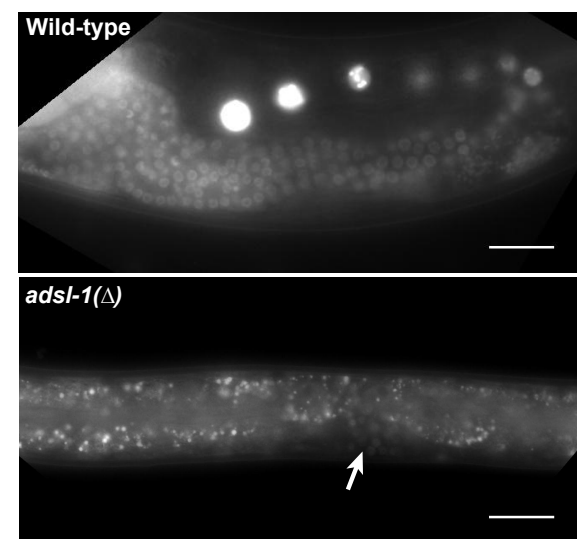

Germline

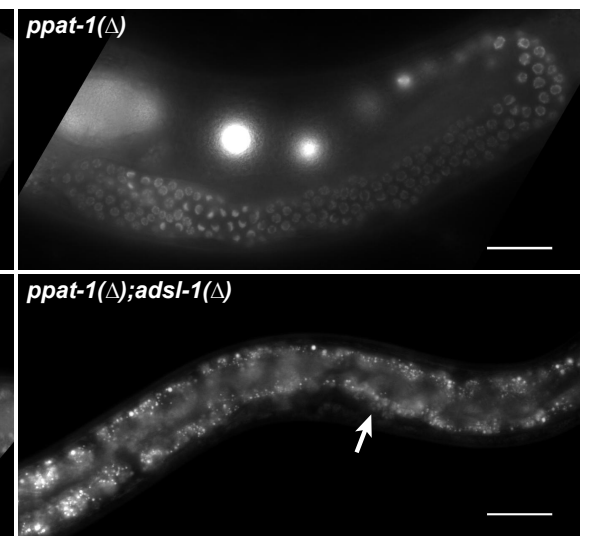

C

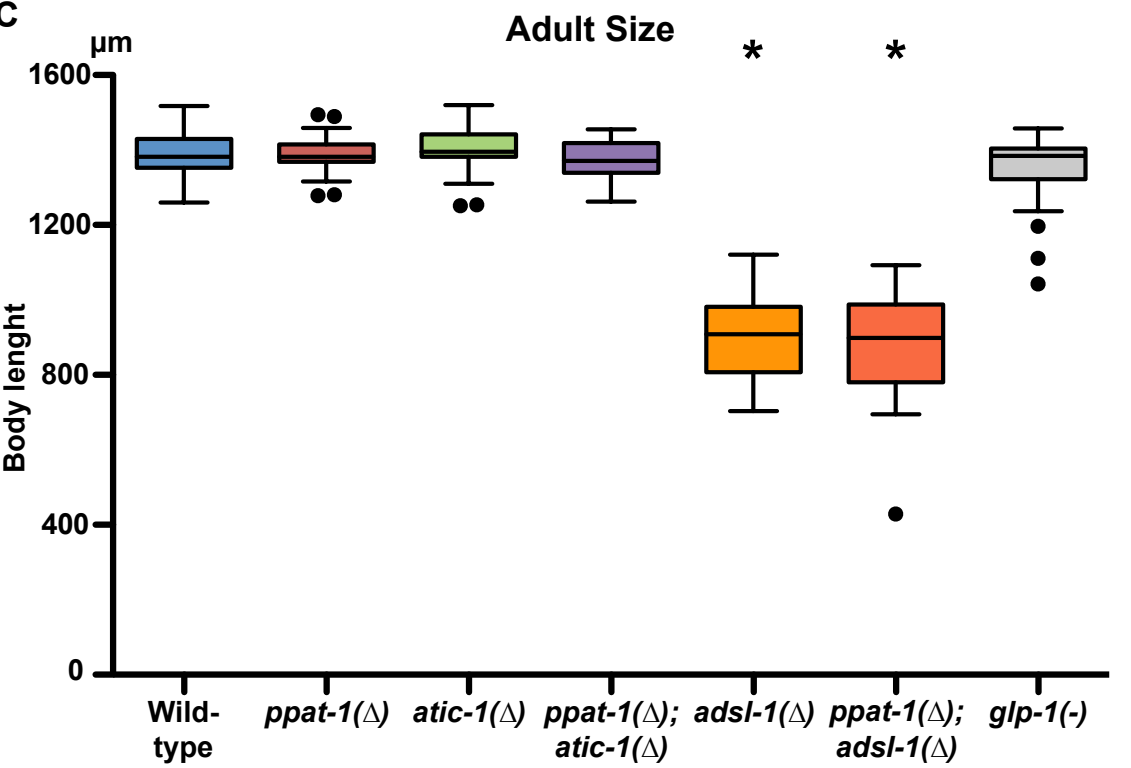

Figure 2 
A

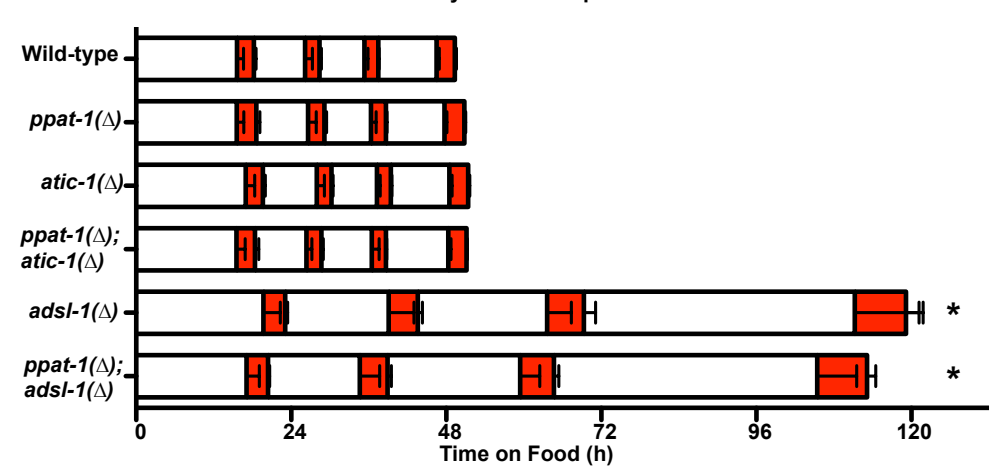

B

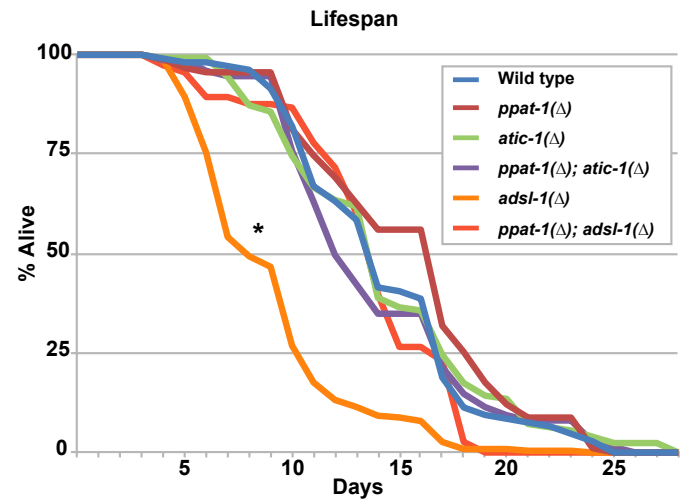

Figure 3

A

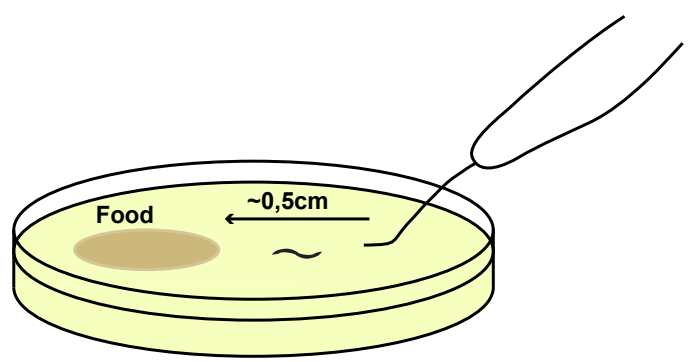

B

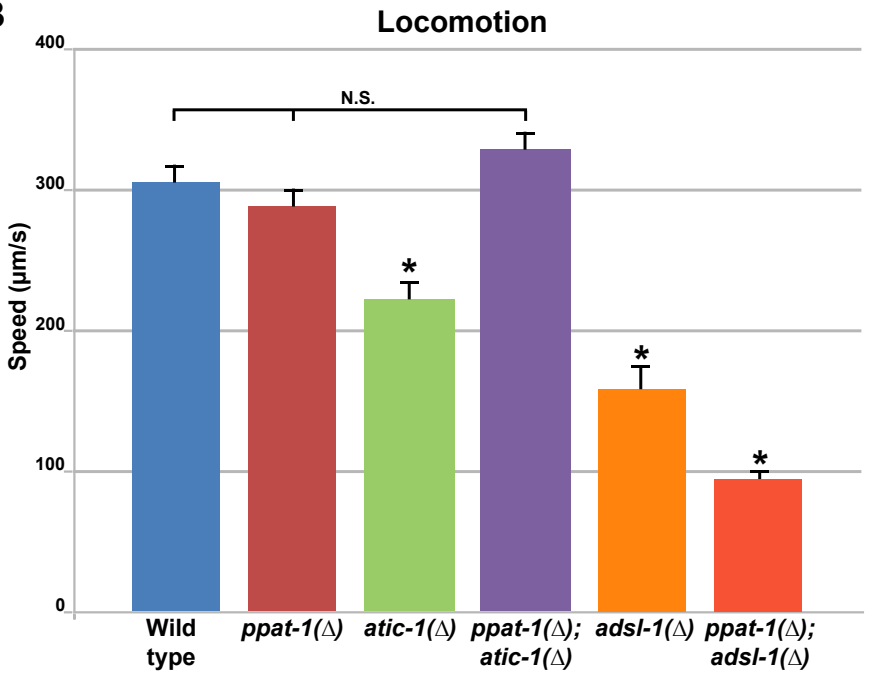

Figure 4

Muscle Myosin
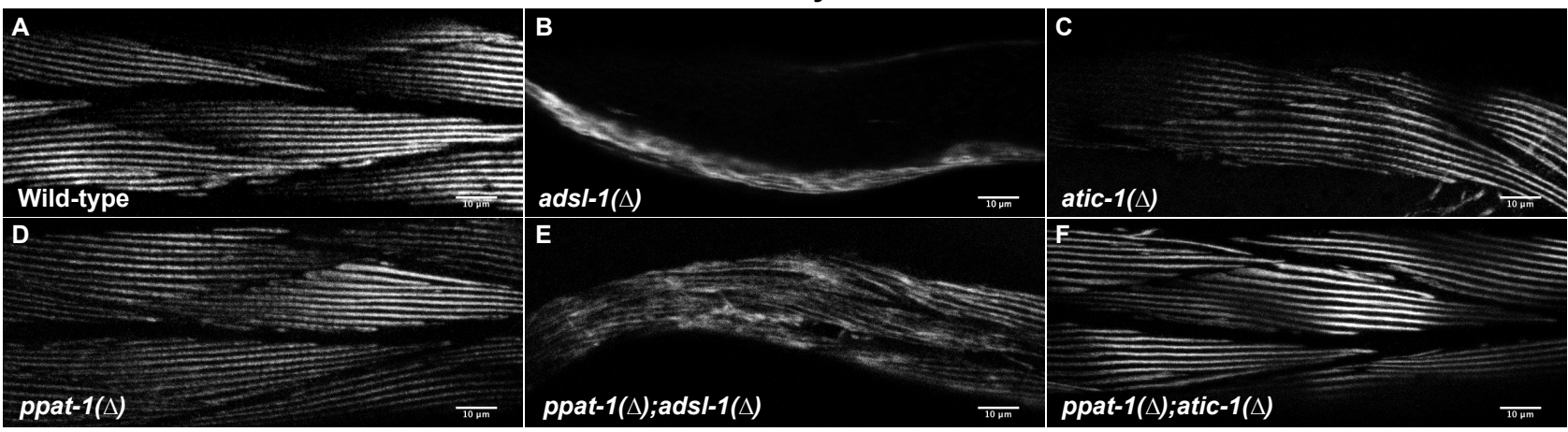

Figure 5 


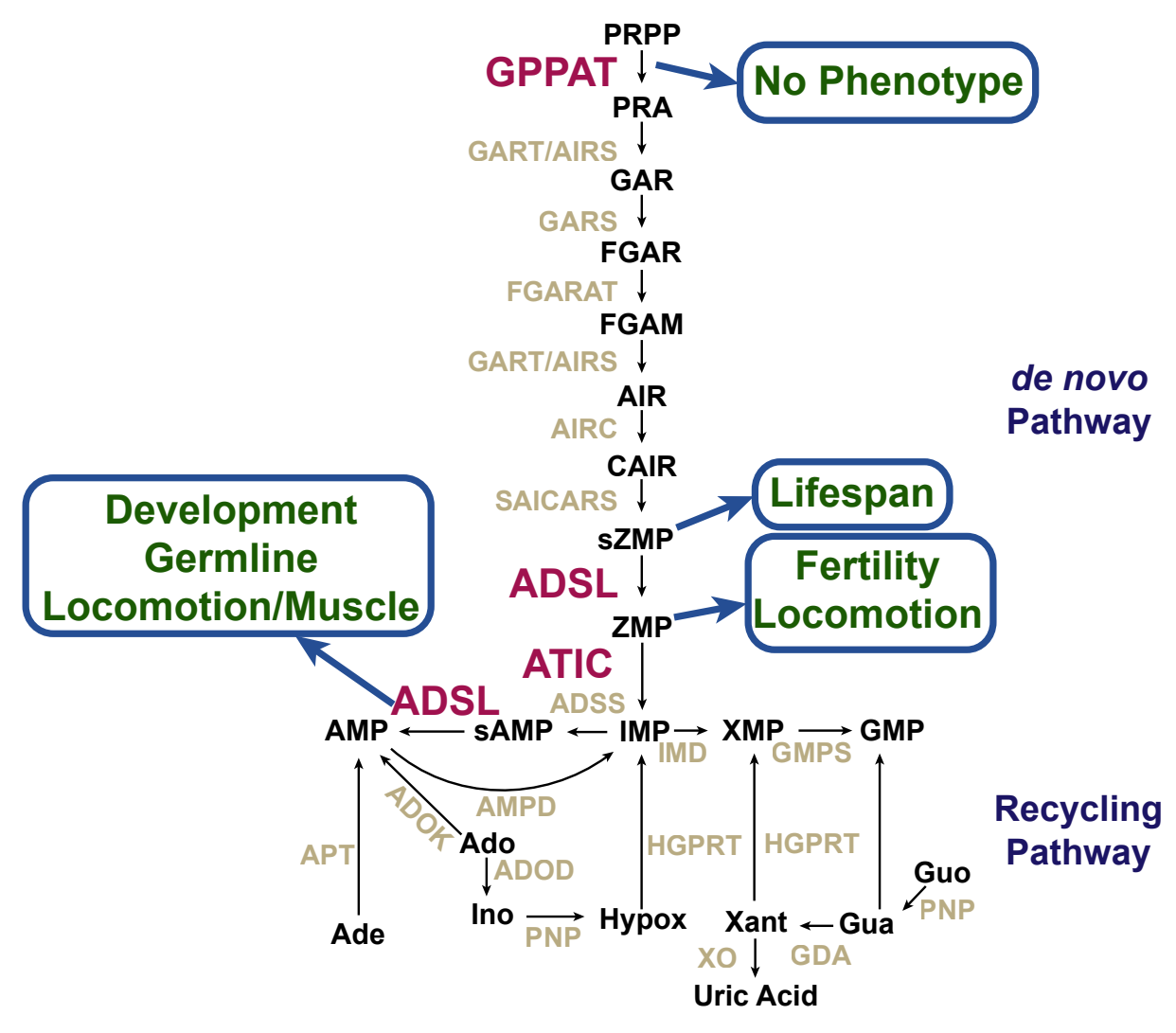

Figure 6 
bioRxiv preprint doi: https://doi.org/10.1101/277640; this version posted April 6, 2018. The copyright holder for this preprint (which was not certified by peer review) is the author/funder, who has granted bioRxiv a license to display the preprint in perpetuity. It is made available under aCC-BY-NC-ND 4.0 International license.
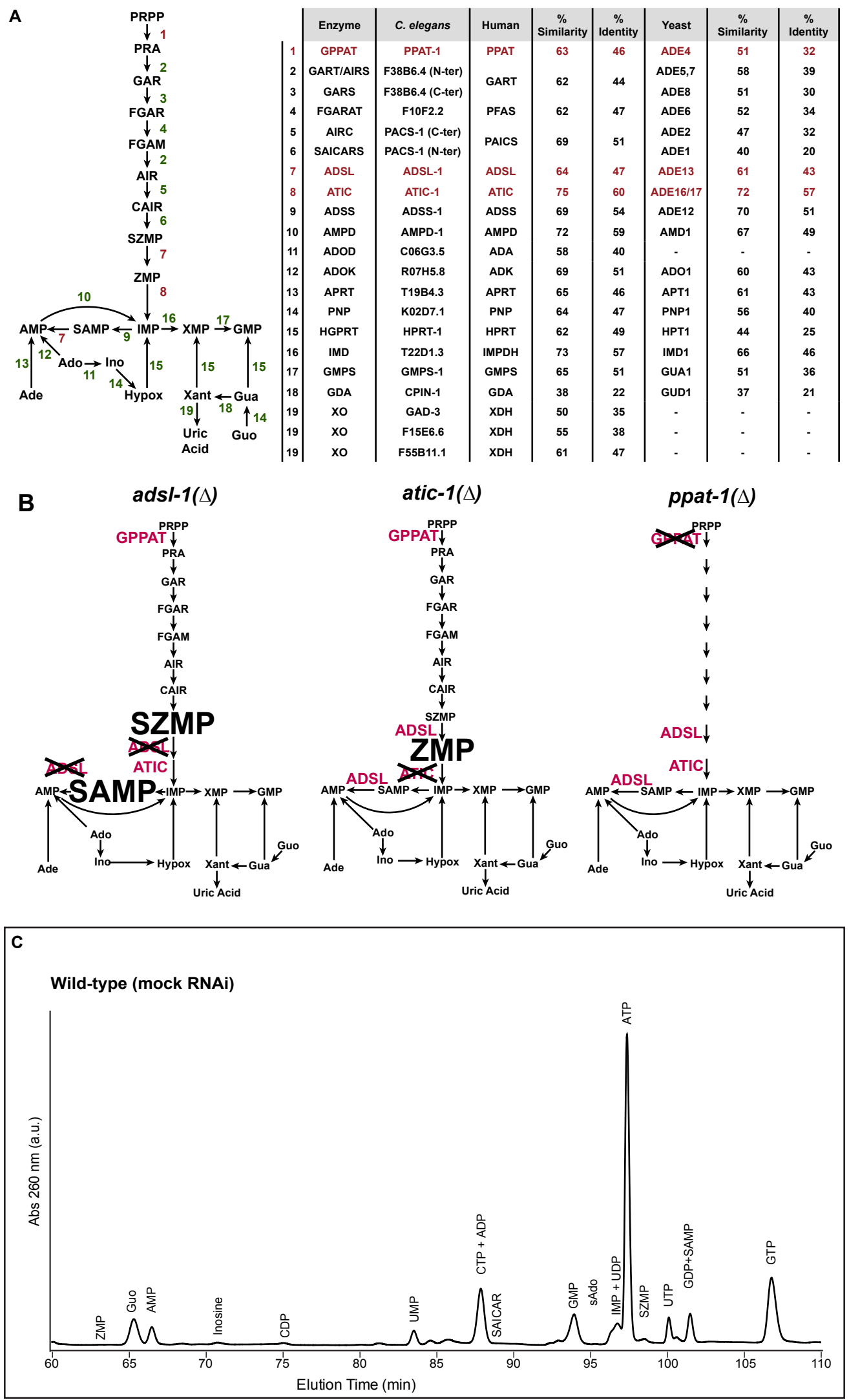

Supplementary Figure 1 


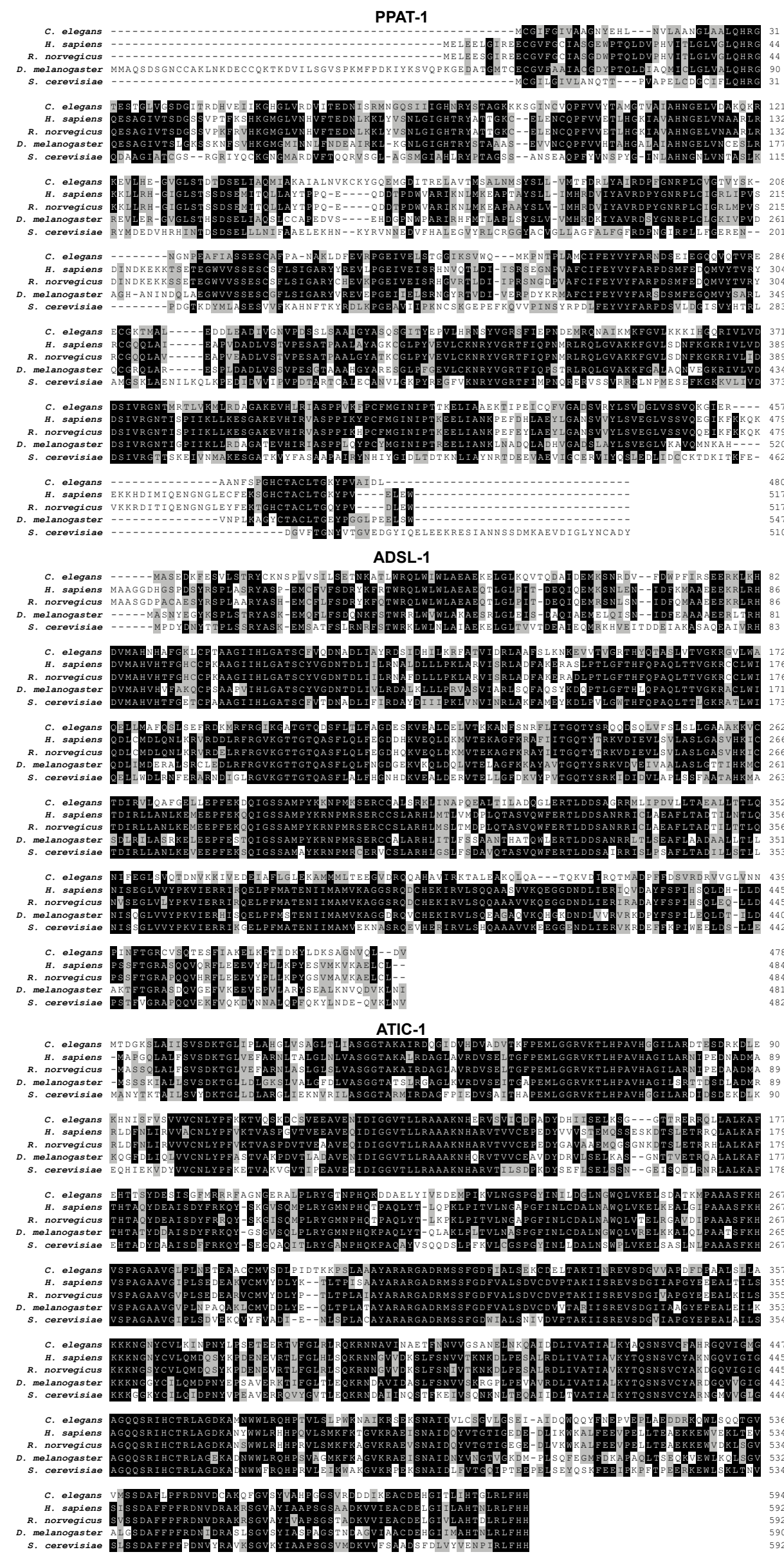

Supplementary Figure 2 
A

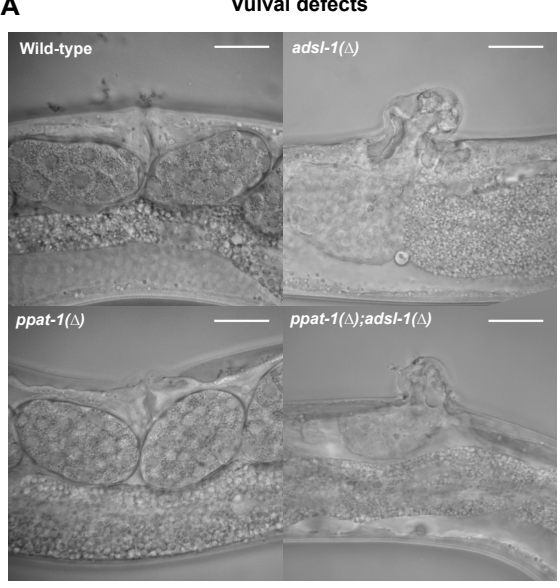

B

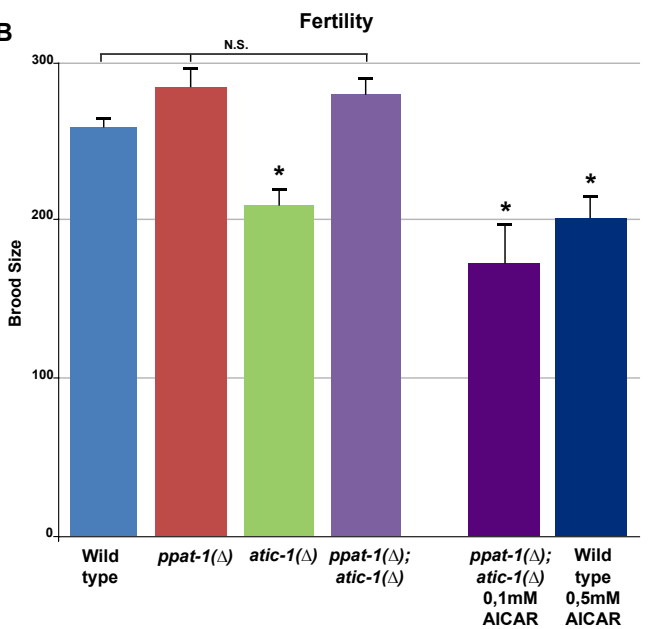

Supplementary Figure 3
C Embryonic lethality

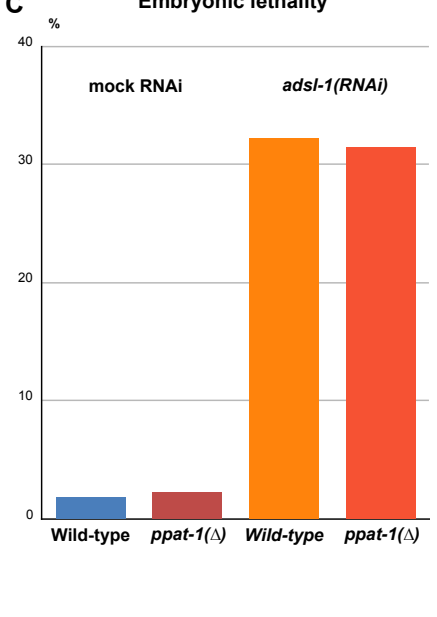

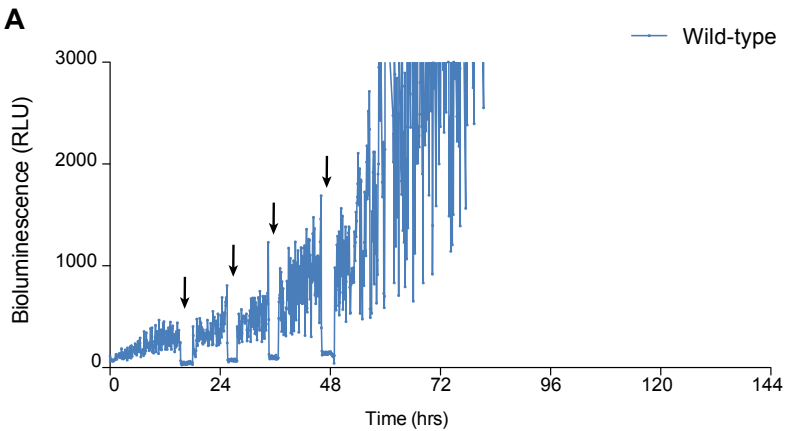

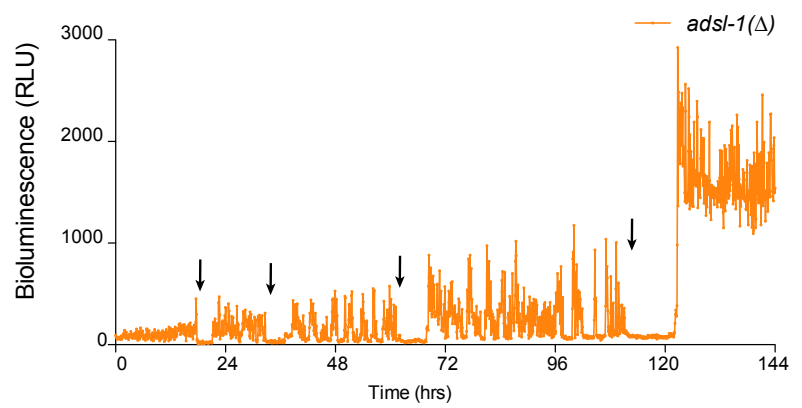

B
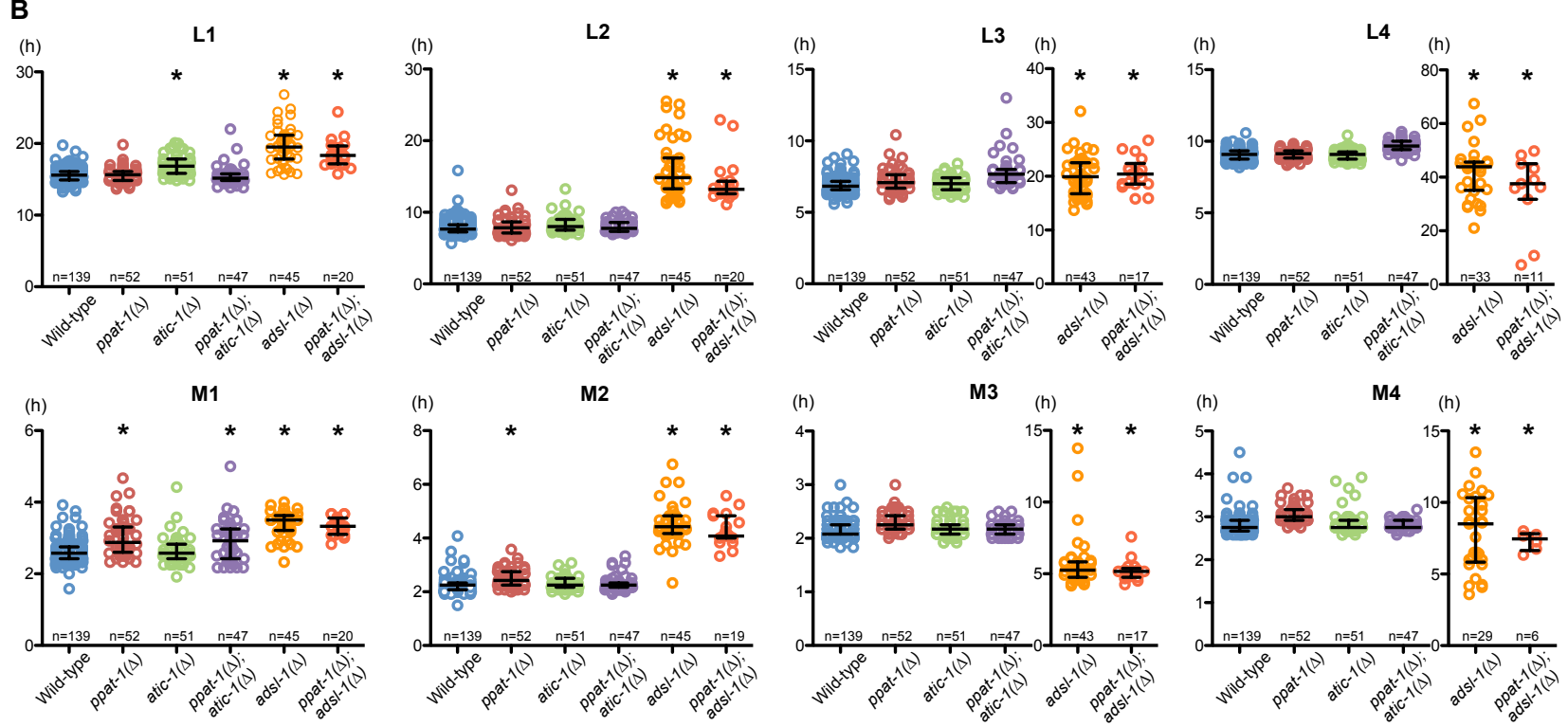

\section{Supplementary Figure 4}


A

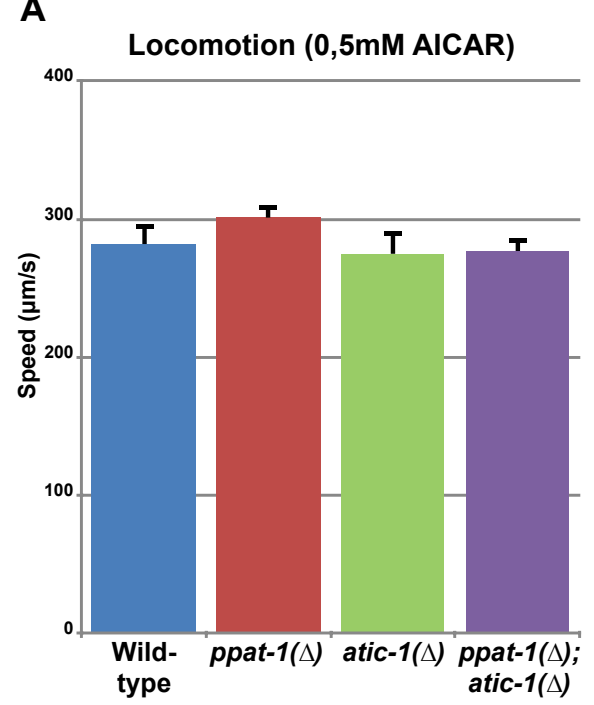

B

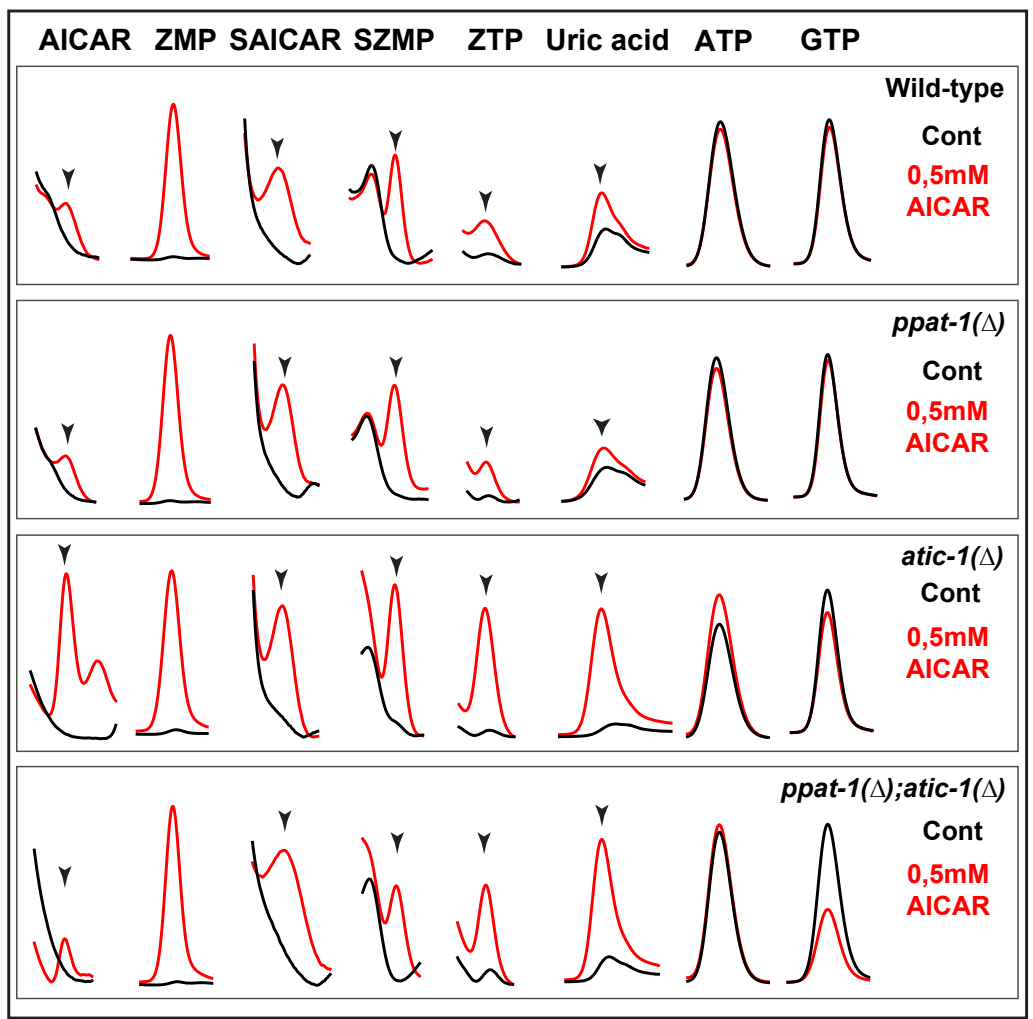

Supplementary Figure 5
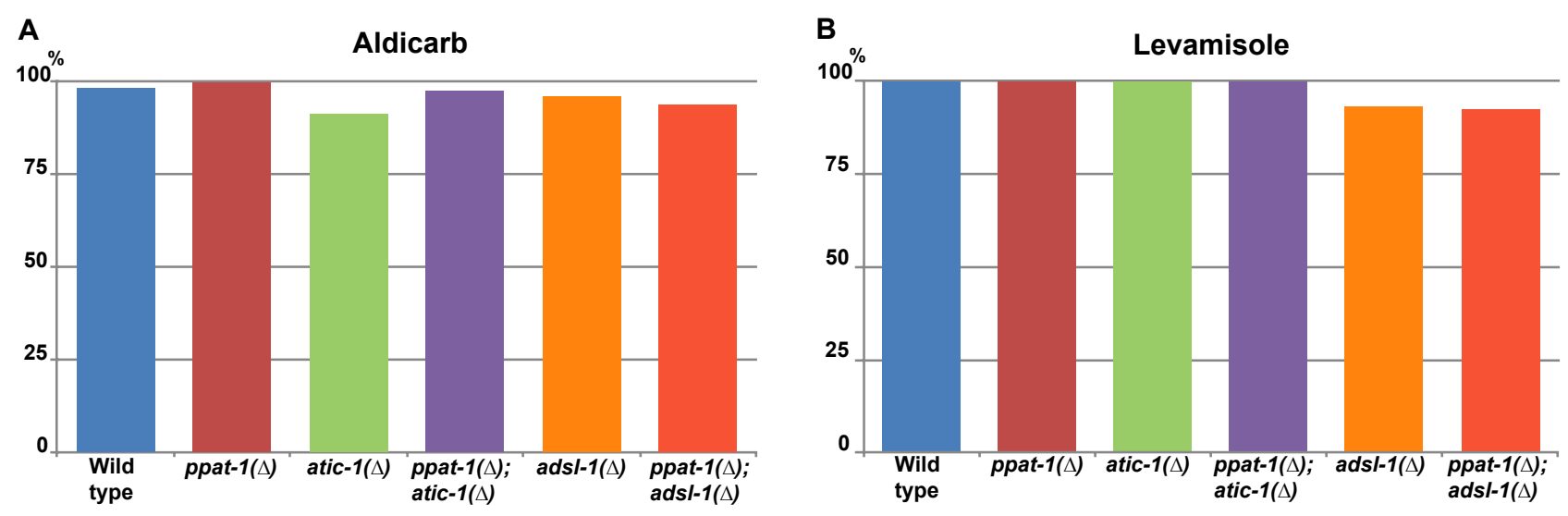

C
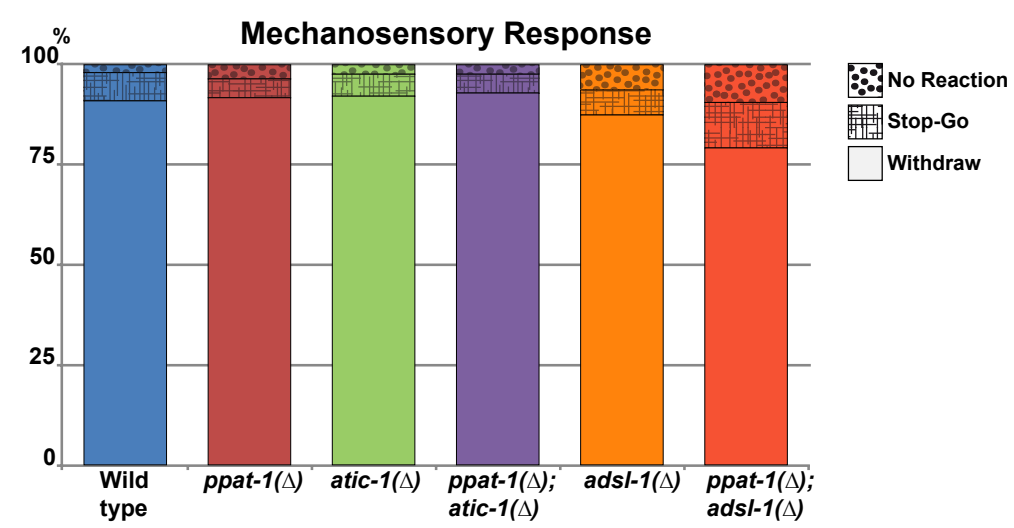